Commun. math. Phys. 31. 209-242 (1973)

(C) by Springer-Verlag 1973

\title{
Tilted Homogeneous Cosmological Models
}

\author{
A. R. King and G. F. R. Ellis \\ Department of Applied Mathematics and Theoretical Physics, University of Cambridge, \\ Cambridge, U.K.
}

Received November 27, 1972; in revised form January 15, 1973

\begin{abstract}
We examine spatially homogeneous cosmological models in which the matter content of space-time is a perfect fluid, and in which the fluid flow vector is not normal to the surfaces of homogeneity. In such universes, the matter may move with non-zero expansion, rotation and shear; we examine the relation between these kinematic quantities and the Bianchi classification of the symmetry group. Detailed characterizations of some of the simplest such universe models are given.
\end{abstract}

\section{Introduction: Covariant Formalism}

In a previous series of papers ([1-3]), exact solutions of Einstein's field equations

$$
R_{a b}-\frac{1}{2} R g_{a b}+\Lambda g_{a b}=T_{a b}
$$

were studied under the assumptions that

(1) the matter takes the "perfect fluid" form:

$$
T_{a b}=\mu u_{a} u_{b}+p\left(g_{a b}+u_{a} u_{b}\right), u_{a} u^{a}=-1, \mu>0, p \geqq 0
$$

where $u^{a}$ is the fluid 4-velocity, $\mu$ the energy density and $p$ the pressure;

(2) space-time is locally invariant under a group of isometries $G_{3}$ simply transitive on spacelike surfaces $S(t)$, i.e. space-time is spatially homogeneous;

(3) the 4-velocity $u^{a}$ is everywhere orthogonal to the homogeneous surfaces $S(t)$.

In this paper, we study a wider class of spatially homogeneous cosmological models: we maintain conditions (1) and (2), but drop (3). This allows a wider variety of behaviour, for when (3) is dropped, the fluid may have non-zero vorticity and acceleration. Further, such a universe may appear to be inhomogeneous to a fundamental observer (e.g. number counts of radio sources or galaxies will look inhomogeneous) even though the space-time and its contents are spatially homogeneous in a strict mathematical sense. Our discussion supplements previous discussions of such universes (see e.g. [4-6]).

The immediate geometrical objects defined in a space-time in which (1) and (2) hold are the surfaces of homogeneity $S(t)$ and the fluid flow 
vector $u^{a}$. The surfaces of homogeneity determine a unique future directed normal vector field $n^{a}, n^{a} n_{a}=-1$, which is geodesic and rotation-free ${ }^{1}$ :

$$
n_{; b}^{a} n^{b}=0, \quad n_{[a} n_{b ; c]}=0 .
$$

Hence $n_{[a ; b]}=0$, which shows there locally exists a function $\tilde{t}$ such that

$$
n_{a}=-\tilde{t}_{, a}, \quad n^{a} n_{a}=-1 \Rightarrow n^{a} \tilde{t}_{, a}=1 .
$$

The surfaces $S(t)$ are then the surfaces $\{\tilde{t}=$ constant $\}$; the time parameter $t$ can be chosen as any $C^{2}$ function $t(\tilde{t})$ such that $d t / d \tilde{t} \neq 0$. All geometrical and physical quantities are invariant on the surfaces $S(t)$; for example, $\mu=\mu(t), p=p(t)$. The metric tensor in these surfaces is $\tilde{h}_{a b} \equiv g_{a b}+n_{a} n_{b}$ (this is the 3-dimensional symmetric projection tensor which projects $n^{a}$ to zero: $\left.\tilde{h}_{a b} n^{b}=0\right)$. For any function $f(t), f_{, a}=(d f / d \tilde{t}) \tilde{t}_{, a}$, so by (1.4)

$$
f=f(t) \Rightarrow f_{, a}=-n_{a}(d f / d \tilde{t}) \Leftrightarrow f_{, a} n^{a}=d f / d \tilde{t}, f_{, a} \tilde{h}_{b}^{a}=0 .
$$

The fluid flow vector $u^{a}$ is uniquely defined as the future directed timelike eigenvector of the Ricci tensor. The projection tensor $h_{a b} \equiv g_{a b}+u_{a} u_{b}$ projects into the instantaneous rest spaces orthogonal to $u^{a}\left(h_{a b} u^{b}=0\right)$; the covariant derivative ${ }_{;} u^{a}$ along the fluid flow lines is denoted by a dot, e.g. the fluid acceleration vector $\dot{u}^{a}$ is $\dot{u}^{a} \equiv u_{a ; b} u^{b}$ (see e.g. $[7,8]$ ).

The relation between $u^{a}$ and $n^{a}$ is determined by

(a) the hyperbolic angle of tilt $\beta$, where

$$
\cosh \beta=-u^{a} n_{a}, \quad \beta(t) \geqq 0,
$$

and by the direction of tilt, specified either by

(b) the direction $\tilde{c}^{a}$ of the projection of $u^{a}$ in the surfaces $S(t)$ :

or by

$$
\tilde{h}_{b}^{a} u^{b}=\sinh \beta \tilde{c}^{a} \Rightarrow \tilde{c}_{a} n^{a}=0, \tilde{c}^{a} \tilde{c}_{a}=1,
$$

(c) the direction $c^{a}$ of $n^{a}$ perpendicular to $u^{a}$ :

Then one has $h_{b}^{a} n^{b}=-\sinh \beta c^{a} \Rightarrow c_{a} u^{a}=0, c_{a} c^{a}=1$.

$$
u^{a}=\cosh \beta n^{a}+\sinh \beta \tilde{c}^{a}, \quad n^{a}=\cosh \beta u^{a}-\sinh \beta c^{a} .
$$

The condition (3) that $u^{a}$ be perpendicular to $S(t)$ is the condition $u^{a}=n^{a} \Leftrightarrow \beta=0$; these were the models studied in [1-3]. We now wish to concentrate our attention on tilted homogeneous cosmological models (or "tilted models"), i.e. those in which (1), (2) hold and

$$
u^{a} \neq n^{a} \Leftrightarrow \beta>0 .
$$

${ }^{1}$ It is rotation-free because it is normal to the surfaces $S$. It is geodesic because it is orthogonal to a family of 3 Killing vector fields $\xi_{v_{a}}(v=1,2,3)$ which are linearly independent at each point; for $\xi_{a} n^{a}=0 \Rightarrow \xi_{v a ; b} n^{a}+\xi_{v a} n_{; b}^{a}=0$. Multiplying by $n^{b}, \xi_{v(a ; b)}=0 \Rightarrow \xi_{v_{a}} n_{; b}^{a} n^{b}=0$ for $v=1,2,3$. Our notation is, as far as possible, identical to that of [1-3].

2 The - sign is included for later convenience (cf. Fig. 1). 


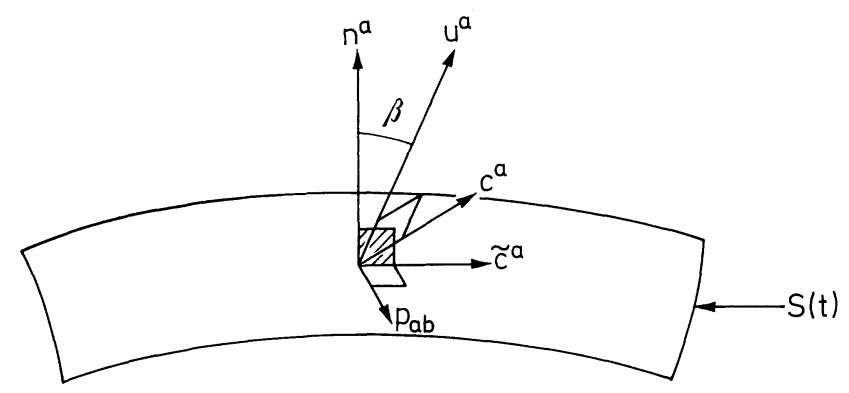

Fig. 1. In a tilted homogeneous cosmology, the normals $n^{a}$ to the surfaces of homogeneity $S(t)$ make a hyperbolic angle $\beta$ with the fluid flow vector $u^{a}$; the unit vectors $c^{a}$, $\tilde{c}^{a}$ are coplanar with $u^{a}, n^{a}$. The 2-surface in $S(t)$ perpendicular to $c^{a}, \tilde{c}^{a}$ is spanned by the tensor $p_{a b}$

When this condition is satisfied, $\tilde{c}^{a}$ and $c^{a}$ are uniquely defined by (1.7), (1.8), and (1.9) expresses the way in which $u^{a}$ is tilted with respect to the surfaces $S$ (see Fig. 1). For some purposes it is useful to think of the factor $\gamma \equiv \cosh \beta$ instead of $\beta$; then $\sinh \beta=\left(\gamma^{2}-1\right)^{\frac{1}{2}}$, and $\gamma$ is the usual relativistic contraction factor for the relative motion (sometimes called the "peculiar velocity") of the fluid with respect to the homogeneous surfaces $\left(u^{a}=\gamma\left(n^{a}+v \tilde{c}^{a}\right)\right.$, where the 3-velocity $\boldsymbol{v}=v \boldsymbol{c}$ is related to $\gamma$ by $\left.\gamma=\left(1-v^{2}\right)^{-\frac{1}{2}}\right)$.

Combining (1.5) with (1.9), we see that

$$
f=f(t) \Rightarrow f^{\cdot} \equiv f_{, a} u^{a}=\cosh \beta d f / d \tilde{t}, \quad f_{, a} h_{b}^{a}=\sinh \beta d f / d \tilde{t} c_{b} .
$$

This shows how tilted models will in general appear inhomogeneous: an observer moving with 4-velocity $u^{a}$ will assign to the (homogeneous) density $\mu(t)$ a spatial gradient of magnitude $\sinh \beta d \mu / d \tilde{t}$, in the direction $c_{a}$. To complete the algebraic relations, note that (1.9) implies

$$
c^{a}=\sinh \beta n^{a}+\cosh \beta \tilde{c}^{a}, \quad \tilde{c}^{a}=-\sinh \beta u^{a}+\cosh \beta c^{a} .
$$

The first covariant derivative of $n^{a}$ is simply [because of (1.3)] the second fundamental form $\tilde{\theta}_{a b}$ of the surfaces $S(t)$ :

$$
n_{a ; b}=\tilde{\theta}_{a b}, \tilde{\theta}_{a b}=\tilde{\theta}_{(a b)}, \tilde{\theta}_{a b} n^{b}=0 .
$$

Its trace is $\tilde{\theta} \equiv \tilde{\theta}^{a}$, and its trace-free part is $\tilde{\sigma}_{a b} \equiv \tilde{\theta}_{a b}-\frac{1}{3} \tilde{\theta} \tilde{h}_{a b}$. By (1.9) the first covariant derivative of $u^{a}$ is, on using (1.5) and (1.12),

$$
u_{a ; b}=-d \beta / d \tilde{t} c_{a} n_{b}+\cosh \beta \tilde{\theta}_{a b}+\sinh \beta \tilde{c}_{a ; b} .
$$

From this, one can obtain expressions for the fluid kinematic quantities ([7, 8]) on using (1.9), (1.12) and (1.13). The acceleration is

$$
\dot{u}_{a}=c_{a} d(\sinh \beta) / d \tilde{t}+\sinh \beta \cosh \beta \tilde{\theta}_{a b} \tilde{c}^{b}+\sinh \beta\left(\tilde{c}_{a}\right),
$$


the expansion $\theta \equiv u_{; a}^{a}$ is

$$
\theta=d(\cosh \beta) / d \tilde{t}+\cosh \beta \tilde{\theta}+\sinh \beta \tilde{c}_{a ; b} \tilde{h}^{a b},
$$

and the vorticity vector $\omega^{a} \equiv \frac{1}{2} \eta^{a b c d} u_{b} u_{c ; d}$ is

$$
\omega^{a}=\sinh \beta \frac{1}{2} \eta^{a b c d} u_{b} \tilde{c}_{c ; d} .
$$

The expressions for the expansion tensor $\theta_{a b}$ and shear tensor $\sigma_{a b}$,

$$
\theta_{a b} \equiv h_{a}{ }^{c} h_{b}{ }^{d} u_{(c ; d)}=u_{(a ; b)}+\dot{u}_{(a} u_{b)}, \sigma_{a b} \equiv \theta_{a b}-\frac{1}{3} \theta h_{a b},
$$

are somewhat more complex. Eqs. (1.15), (1.17) immediately show how the fluid in a tilted model can have non-zero acceleration and vorticity; from (1.17), the projection of $\omega^{a}$ in the $\tilde{c}^{a}$ direction is

$$
\tilde{c}_{a} \omega^{a}=\sinh \beta \cosh \beta \frac{1}{2} \eta^{a b c d} \tilde{c}_{a} n_{b} \tilde{c}_{c ; d}=-\operatorname{coth} \beta n_{a} \omega^{a} .
$$

From the second expression, one sees that the vector $\omega^{a}$ lies in the surfaces of homogeneity if and only if it is perpendicular to the tilt directions $\tilde{c}^{a}, c^{a}$. One may alternatively give the vorticity by specifying the vorticity tensor

$$
\omega_{a b} \equiv \eta_{a b c d} \omega^{c} u^{d}=u_{[a ; b]}+\dot{u}_{[a} u_{b]} \text {. }
$$

Relations (1.18), (1.20) show that $\sigma^{a}{ }_{a}=0, \sigma_{a b}=\sigma_{(a b)}, \omega_{a b}=\omega_{[a b]}, \sigma_{a b} u^{b}=0$ $=\omega_{a b} u^{b}$; from (1.9) one has then

$$
\sigma_{a b} n^{b}=-\sigma_{a b} \tilde{c}^{b} \tanh \beta, \quad \omega_{a b} n^{b}=-\omega_{a b} \tilde{c}^{b} \tanh \beta .
$$

It will be convenient later to consider average lengths $l(t), \tilde{l}(t)$ defined (up to a multiplicative factor) by $l l^{-1}=\frac{1}{3} \theta(t), d \tilde{l} / d \tilde{t} \tilde{l}^{-1}=\frac{1}{3} \tilde{\theta}(t)$. They represent respectively the volume behaviour of the fluid congruence and the normal congruence (see $[7,8]$ ).

The components $u_{a} T_{; b}^{a b}=0$ and $h_{a}^{c} T_{; b}^{a b}=0$ of the conservation equations $T^{a b}{ }_{; b}=0$ for the perfect fluid (1.2) take the form

$$
\mu+(\mu+p) \theta=0, \quad h_{a}^{b} p_{, b}+(\mu+p) \dot{u}_{a}=0 .
$$

Defining functions $w(t), r(t)$ from $\mu(t), p(t)$ by

$$
w(t) \equiv \exp \int_{t_{0}}^{t} \frac{d \mu / d t}{(\mu+p)} d t, \quad r(t) \equiv \exp \int_{t_{0}}^{t} \frac{d p / d t}{(\mu+p)} d t,
$$

Eqs. (1.22) become [using (1.11)]

$$
\cosh \beta d(\log w) / d \tilde{t}+\theta=0, \quad \sinh \beta d(\log r) / d \tilde{t} c_{a}+\dot{u}_{a}=0 ;
$$

and one can combine Eqs. (1.22) to give the relation

$$
\dot{u}_{a}=\tanh \beta d p / d \mu \theta c_{a} .
$$


This shows that in an expanding tilted universe, either $\dot{u}_{a} \neq 0$ or $d p / d \mu=0$. Clearly $\dot{u}_{a}=0$ if $p=0$; for realistic equations of state with $p \neq 0$, an expanding tilted universe will have non-zero acceleration. Further, (1.25) shows that $\dot{u}_{a}$ is parallel to $c_{a}$. It now follows from (1.20) and (1.14) that

$$
\tilde{h}_{a}{ }^{c} \tilde{h}_{b}{ }^{d} \omega_{c d}=\tilde{h}_{a}{ }^{c} \tilde{h}_{b}{ }^{d} u_{[c ; d]}=\sinh \beta \tilde{h}_{a}{ }^{c} \tilde{h}_{b}{ }^{d} \tilde{c}_{[c ; d]}
$$

and from (1.18), (1.25) and (1.14) that

$$
\begin{aligned}
\tilde{h}_{a}{ }^{c} \tilde{h}_{b}{ }^{d} \sigma_{c d}= & \cosh \beta \tilde{\theta}_{a b}-\frac{1}{3} \theta \tilde{h}_{a b} \\
& +\sinh \beta \tilde{h}_{a}{ }^{c} \tilde{h}_{b}{ }^{d} \tilde{c}_{(c ; d)}-\theta \sinh ^{2} \beta\left(\frac{1}{3}-\frac{d p}{d \mu}\right) \tilde{c}_{a} \tilde{c}_{b} ;
\end{aligned}
$$

by (1.21), these components are sufficient to determine $\omega_{a b}$ and $\sigma_{a b}$ (note that $\tilde{h}_{a}{ }^{c} \tilde{h}_{b}{ }^{d} \sigma_{c d}$ is not necessarily trace-free; in fact, $\tilde{h}^{c d} \sigma_{c d}=n^{c} n^{d} \sigma_{c d}$ $=\tanh ^{2} \beta \sigma_{a b} \tilde{c}^{a} \tilde{c}^{b}$ ).

Combining (1.24) with (1.15), (1.16), the conservation equations take the form

$$
\begin{aligned}
& \frac{d}{d t} \log \left(w \tilde{l}^{3} \cosh \beta\right)+\tanh \beta \tilde{c}_{a ; b} \tilde{h}^{a b}=0, \\
& \frac{d}{d \tilde{t}} \log (r \sinh \beta) c_{a}+\left(\tilde{c}_{a}\right)^{\cdot}+\cosh \beta \tilde{\theta}_{a b} \tilde{c}^{b}=0 .
\end{aligned}
$$

To clarify the meaning of these equations, we introduce the projection tensor

$$
p_{a b} \equiv h_{a b}-c_{a} c_{b}=\tilde{h}_{a b}-\tilde{c}_{a} \tilde{c}_{b}
$$

which projects at each point into the 2 -space perpendicular to $u^{a}$ and $n^{a}$ (this is uniquely defined when $\beta>0$ ). Multiplying (1.29) by $\tilde{c}^{a}$ one obtains

$$
\frac{d}{d t} \log (r \sinh \beta)+\tilde{c}^{a} \tilde{\theta}_{a b} \tilde{c}^{b}=0
$$

while multiplying by $p_{a}{ }^{b}$ shows that

$$
p_{a}{ }^{b}\left(\tilde{c}_{b}\right)^{\cdot}+\cosh \beta p_{a}{ }^{b} \tilde{\theta}_{b c} \tilde{c}^{c}=0
$$

or equivalently, combining (1.29) and (1.31),

$$
\left(\tilde{c}_{a}\right)^{\cdot}=-\cosh \beta \tilde{\theta}_{a b} \tilde{c}^{b}+c_{a} \tilde{c}^{b} \tilde{\theta}_{b c} \tilde{c}^{c} .
$$

Thus two of the conservation equations determine the rate of rotation of $\tilde{c}_{a}$ along the fluid flow lines in a way which is independent of the equation of state of the fluid. The other two, namely (1.28) and (1.31), determine the evolution of the fluid quantities and the angle $\beta$, once an equation of state $p=p(\mu)$ is specified ${ }^{3}$. The form of $(1.31)$ shows that $\beta$ is either zero or non-zero for all $t$, i.e. a tilted universe model stays tilted.

${ }^{3}$ One can re-express (1.28) in the form $\left(w l^{3}\right)^{\circ}=0$, which shows that $w l^{3}$ stays constant along the fluid flow lines. Note that by (1.23), one can normalize $r, w$ so that $r w=\mu+p$. 
Finally we note that in a tilted universe model with perfect fluid stress tensor (1.2), the stress tensor takes the form

$$
T_{a b}=\tilde{\mu} n_{a} n_{b}+\tilde{p} \tilde{h}_{a b}+2 \tilde{q}_{(a} n_{b)}+\tilde{\pi}_{a b}
$$

when decomposed with respect to the vector $n^{a}$ (cf. [7, 8]), where

$$
\begin{array}{cl}
\tilde{\mu}=\mu \cosh ^{2} \beta+p \sinh ^{2} \beta, & \tilde{q}_{a}=(\mu+p) \sinh \beta \cosh \beta \tilde{c}_{a}, \\
\tilde{p}=p+\frac{1}{3}(\mu+p) \sinh ^{2} \beta, & \tilde{\pi}_{a b}=(\mu+p) \sinh ^{2} \beta\left(\tilde{c}_{a} \tilde{c}_{b}-\frac{1}{3} \tilde{h}_{a b}\right) .
\end{array}
$$

By (1.2), $(\mu+p)>0$, so (1.33) shows

$$
\beta>0 \Rightarrow \tilde{q}_{a} \neq 0, \quad \tilde{\pi}_{a b} \neq 0,
$$

i.e. in this frame, the stress tensor of a tilted cosmological model is that of an imperfect fluid with particular equations of state. Using (1.33), one can write down the components $n_{a} T_{; b}^{a b}=0$ and $\tilde{h}_{d}^{a} T_{; b}^{d b}=0$ of the conservation equations: they are

$$
\begin{aligned}
& \frac{d}{d \tilde{t}}\left(\mu \cosh ^{2} \beta+p \sinh ^{2} \beta\right) \\
& +(\mu+p)\left(\cosh ^{2} \beta \tilde{\theta}+\sinh ^{2} \beta \tilde{\theta}_{a b} \tilde{c}^{a} \tilde{c}^{b}+\sinh \beta \cosh \beta \tilde{c}_{; b}^{b}\right)=0,
\end{aligned}
$$

governing the rate of change of the total energy density $\tilde{\mu}$, and

$$
\begin{aligned}
\left((\mu+p) \sinh \beta \cosh \beta \tilde{c}_{a}\right)_{; b} n^{b} & +(\mu+p)\left(\sinh \beta \cosh \beta\left(\tilde{\theta}_{a}^{b}+\tilde{\theta} \tilde{h}_{a}^{b}\right) \tilde{c}_{b}\right. \\
& \left.+\sinh ^{2} \beta\left(\tilde{c}_{a} \tilde{c}_{; b}^{b}+\tilde{h}_{a}{ }^{d} \tilde{c}_{d ; b} \tilde{c}^{b}\right)\right)=0,
\end{aligned}
$$

governing the rate of change of the momentum $\tilde{q}_{a}$ of the matter. These can be rewritten in the form (1.28) (relating $d \mu / d \tilde{t}$ and $d \beta / d \tilde{t}$ ), (1.31) (relating $d p / d \tilde{t}$ and $d \beta / d \tilde{t})$, and

$$
\tilde{c}^{d}{ }_{; b} n^{b}+\tilde{\theta}_{b}^{d} \tilde{c}^{b}-\tilde{c}^{d} \tilde{\theta}_{a b} \tilde{c}^{a} \tilde{c}^{b}+\tanh \beta \tilde{h}_{c}^{d} \tilde{c}^{c}{ }_{; b} \tilde{c}^{b}=0
$$

[which is equivalent to (1.32)]. One sees from these relations that if the pressure is constant (in particular, if it vanishes) the rate of change of $\beta$ is determined by $\tilde{\theta}_{a b} \tilde{c}^{a} \tilde{c}^{b}$ alone; and that one could instead of specifying an equation of state $p(\mu)$, specify $\beta$ as a function of $t$ (e.g. specify that $\beta=$ constant $>0$ ), Eqs. (1.31) and (1.28) then determining an implicit equation of state which will lead to the specified $\beta(t)$.

\section{Tetrad Description}

To examine the classification of the spaces according to the group of isometries acting, it is convenient to introduce various tetrads, i.e. various bases of vectors $\left\{\boldsymbol{E}_{a}\right\}, a=0,1,2,3$. We denote the derivatives of any function $f$ in the basis vector directions by $\partial_{a} f$, so if $E_{a}{ }^{i}$ are the 
components of the vectors $\left\{\boldsymbol{E}_{a}\right\}$ in a local coordinate frame, $\partial_{a} f$ $=E_{a}{ }^{i} \partial f / \partial x^{i}$. The inverse matrix will be denoted by $E^{a}{ }_{i}$, so $E^{a}{ }_{i} E_{a}{ }^{j}=\delta_{i}{ }^{j}$. Any tensor with coordinate components $T^{i \ldots j}{ }_{k \ldots l}$ has tetrad components $T^{a \ldots b}{ }_{c \ldots d}$ defined by $T^{a \ldots b}{ }_{c \ldots d}=E^{a}{ }_{i} \ldots E^{b}{ }_{j} T^{i \ldots j}{ }_{k \ldots l} E_{c}{ }^{k} \ldots E_{d}{ }^{l}$. Thus the metric tensor components $g_{a b}, g^{a b}$ are defined by

$$
g_{a b}=\boldsymbol{E}_{a} \cdot \boldsymbol{E}_{b}=E_{a}^{i} E_{b}{ }^{j} g_{i j}, \quad g^{a b}=E^{a}{ }_{i} E^{b}{ }_{j} g^{i j} \Rightarrow g^{a b} g_{b c}=\delta^{a}{ }_{c},
$$

which imply the inverse relations

$$
g_{i j}=E_{i}^{a} E_{j}^{b} g_{a b}, \quad g^{i j}=E_{a}{ }^{i} E_{b}^{j} g^{a b} .
$$

The differential properties of the basis may be characterized by the rotation coefficients $\Gamma_{a b c}$ or $\Gamma_{b c}^{a}$, where

$$
\Gamma_{a b c}=E_{a}^{i} E_{c i ; j} E_{b}^{j}, \quad \Gamma_{b c}^{a}=g^{a d} \Gamma_{d b c} .
$$

Thus $\Gamma_{a b c}$ is simply the $\boldsymbol{E}_{a}$-component of the covariant derivative in the $\boldsymbol{E}_{b}$-direction of the $\boldsymbol{E}_{c}$-vector. Alternatively one may consider the basis vector commutators. For any vector fields $\boldsymbol{X}, \boldsymbol{Y}$, the commutator $[\boldsymbol{X}, \boldsymbol{Y}]$ is the vector field with components

$$
[X, Y]^{i}=Y_{; j}^{i} X^{j}-X_{; j}^{i} Y^{j}=Y^{i}{ }_{j} X^{j}-X_{, j}^{i} Y^{j} .
$$

It may also be characterized as the Lie derivative $L_{\mathbf{X}} \boldsymbol{Y}$ of these vector fields $([9,10]): L_{\boldsymbol{X}} \boldsymbol{Y}=[\boldsymbol{X}, \boldsymbol{Y}]$. Applying (2.3) to the basis vectors $\left\{\boldsymbol{E}_{a}\right\}$, we define commutation functions $\gamma^{a}{ }_{b c}, \gamma_{a b c}$ by

$$
\left[\boldsymbol{E}_{a}, \boldsymbol{E}_{b}\right]=\gamma_{a b}^{c} \boldsymbol{E}_{c}, \gamma_{a b}^{c}=\gamma_{[a b]}^{c}, \gamma_{c a b}=g_{c d} \gamma_{a b}^{d} .
$$

It follows from (2.2) that

$$
\gamma_{b c}^{a}=\Gamma_{b c}^{a}-\Gamma_{c b}^{a} \Leftrightarrow \gamma_{a b c}=\Gamma_{a b c}-\Gamma_{a c b} ;
$$

then the relations $g_{i j ; k}=0$ show that

$$
\begin{aligned}
\partial_{b} g_{a c} & =\Gamma_{a b c}+\Gamma_{c b a} \Leftrightarrow \Gamma_{a b c} \\
& =\frac{1}{2}\left(\partial_{b} g_{c a}+\partial_{c} g_{a b}-\partial_{a} g_{b c}\right)+\frac{1}{2}\left(\gamma_{a b c}+\gamma_{c a b}-\gamma_{b c a}\right) .
\end{aligned}
$$

Taking the tetrad components of the curvature tensor, which we define by the Ricci identity

$$
V_{; c d}^{b}-V_{; d c}^{b}=-R^{b}{ }_{e c d} V^{e},
$$

and contracting, one obtains the field equations (1.1) in the tetrad form

$$
\partial_{d} \Gamma_{c b}^{c}-\partial_{c} \Gamma_{d b}^{c}-\Gamma_{c s}^{c} \Gamma_{d b}^{s}+\Gamma_{c b}^{s} \Gamma_{s d}^{c}=T_{d b}-\frac{1}{2} T g_{b d}+\Lambda g_{b d} .
$$

The identities $R_{[b c d]}^{a}=0$ are equivalent to the Jacobi identities

$$
\begin{gathered}
{\left[\boldsymbol{E}_{b},\left[\boldsymbol{E}_{c}, \boldsymbol{E}_{d}\right]\right]+\left[\boldsymbol{E}_{d},\left[\boldsymbol{E}_{b}, \boldsymbol{E}_{c}\right]\right]+\left[\boldsymbol{E}_{c},\left[\boldsymbol{E}_{d}, \boldsymbol{E}_{b}\right]\right]} \\
=0 \Leftrightarrow \partial_{[d} \gamma_{b c]}^{f}+\gamma_{[d b}^{s} \gamma_{c] s}^{f}=0 .
\end{gathered}
$$


To express the spatial homogeneity of the universe models, we restrict the bases we consider to those which are invariant under the group of isometries. One way of obtaining such a basis is to choose the basis arbitrarily at one point in each surface $S(t)$, and then to move it over the surfaces $S(t)$ by the action of the group. It then follows (cf. [1]) that the $\gamma^{a}{ }_{b c}$ are functions only of $t$; and Killing's equations then show that the $g_{a b}$ are functions only of $t$. Hence for such bases,

$$
g_{a b}=g_{a b}(t), \gamma_{a b}^{c}=\gamma_{a b}^{c}(t) \Rightarrow \partial_{c} g_{a b}=\partial_{c} g_{a b}(t), \Gamma_{a b c}=\Gamma_{a b c}(t) .
$$

Clearly the bases are determined up to a Lorentz transformation whose coefficients are functions of $t$ only; Eq. (2.10) expresses the homogeneity of space-time on the surfaces $S(t)$.

To classify the groups of isometries, we consider a sub-basis of vectors $\left\{\tilde{\boldsymbol{E}}_{\mu}\right\}$ which spans the surface $S(t)$ at each point, and is invariant under the group. Their commutators will be linear combinations of themselves (this follows immediately from their geometric interpretation as Lie derivatives), i.e. $\left[\tilde{\boldsymbol{E}}_{\kappa}, \tilde{\boldsymbol{E}}_{\mu}\right]=\tilde{\gamma}^{v}{ }_{\kappa \mu} \tilde{\boldsymbol{E}}_{v}$ where $\tilde{\gamma}^{\kappa}{ }_{\mu \nu}=\tilde{\gamma}^{\kappa}{ }_{\mu \nu}(t)$. Following the procedure of Schücking, Kundt and Behr as in [1], we decompose the $\tilde{\gamma}^{\kappa}{ }_{\mu \nu}$ into a symmetric relative tensor $n^{\mu \nu}$ and a relative vector $a_{\kappa}$ :

$$
\left[\tilde{\boldsymbol{E}}_{\kappa}, \tilde{\boldsymbol{E}}_{\mu}\right]=\tilde{\gamma}^{v}{ }_{\kappa \mu} \tilde{\boldsymbol{E}}_{v}=\left(\varepsilon_{\kappa \mu \tau} n^{\tau \nu}+\delta^{v}{ }_{\mu} a_{\kappa}-\delta^{v}{ }_{\kappa} a_{\mu}\right) \tilde{\boldsymbol{E}}_{v}
$$

where $n^{\mu v}=n^{\mu v}(t)=n^{(\mu v)}, a_{\kappa}=a_{\kappa}(t)$. The characteristic equation for $n^{\mu v}$ is $-\lambda^{3}+\lambda^{2} n+\lambda N+\operatorname{det} n^{\alpha \beta}=0$, where $n \equiv n_{\alpha}^{\alpha}, N \equiv \frac{1}{2}\left(n^{\alpha \beta} n_{\alpha \beta}-n^{2}\right)$. The Jacobi identities (2.9) for the vectors $\left\{\tilde{\boldsymbol{E}}_{\mu}\right\}$ are

$$
n^{\alpha \beta} a_{\beta}=0 .
$$

One can classify the solutions of this equation as in Table 1 , giving the Bianchi-Schücking-Behr classification of group types (cf. [1]). As the group action is analytic, the group type remains constant (i.e. is independent of $t$ ).

Table 1. Classification of groups into classes A and B, and group types I to IX. The parameter $h$ is defined by $h=-a^{2} / N$, where $a^{2} \equiv a^{\kappa} a_{\kappa}$; Bianchi type III is type $\mathrm{VI}_{h}$ with $h=-1$

\begin{tabular}{llll}
\hline Group class & \multicolumn{3}{c}{ Group type } \\
\hline Class $\mathrm{A} \Leftrightarrow a_{\kappa}=0$ & $\operatorname{det} n^{\alpha \beta} \neq 0$ & $\mathrm{IX}: \operatorname{det} n^{\alpha \beta}>0$ & $\mathrm{VIII}: \operatorname{det} n^{\alpha \beta}<0$ \\
& $\operatorname{det} n^{\alpha \beta}=0$ & $\mathrm{VI}_{0}: N>0$ & $\mathrm{VII}_{0}: N<0$ \\
& & $\mathrm{II}: N=0, n>0$ & $\mathrm{I}: \quad N=n=0$ \\
Class $\mathrm{B} \Leftrightarrow a_{\kappa} \neq 0$ & $\operatorname{det} n^{\alpha \beta}=0$ & $\mathrm{VI}_{h}: N>0$ & $\mathrm{VII}_{h}: N<0$ \\
& & $\mathrm{IV}: N=0, n>0$ & $\mathrm{~V}: N=n=0$ \\
\hline
\end{tabular}


One way of completing a triad $\left\{\tilde{\boldsymbol{E}}_{\mu}\right\}$ to obtain a complete basis of vectors is to add the normal vector $\boldsymbol{n}$, making a "normal basis" $\left\{\boldsymbol{E}_{a}\right\}$ $=\left\{\boldsymbol{n}, \tilde{\boldsymbol{E}}_{\mu}\right\}$. When $\beta=0$, there is no other compelling choice, but when $\beta>0$ the choice is not so obvious; this basis is closely related to the symmetry properties of the space-time, but not to the properties of the fluid. Two alternatives are to use a "tilted basis" $\left\{\boldsymbol{E}_{a}\right\}=\left\{r^{-1} \boldsymbol{u}, \tilde{\boldsymbol{E}}_{\mu}\right\}$, where the timelike vector is parallel to $\boldsymbol{u}$ and so is not orthogonal to the spacelike vectors, or to use a "fluid basis" $\left\{\boldsymbol{E}_{a}\right\}=\left\{r^{-1} \boldsymbol{u}, \boldsymbol{E}_{\mu}\right\}$, where the vectors $\left\{\boldsymbol{E}_{\mu}\right\}$ are orthogonal to $\boldsymbol{u}$ and so do not span the surfaces $S(t)$. The inclusion of the factor $r(t)$ [defined by (1.23)] is in order to simplify the tetrad form of the conservation equations. A particular choice of the time coordinate $t$ can conveniently be associated with these two bases, by defining $t(\tilde{t})$ to satisfy the relations

$$
t_{, a} E_{0}{ }^{a}=t_{, a}\left(r^{-1} u^{a}\right)=1 \Leftrightarrow d t / d \tilde{t}=r(\cosh \beta)^{-1} .
$$

We shall restrict the bases we consider to ones in which the triads $\left\{\tilde{\boldsymbol{E}}_{\mu}\right\},\left\{\boldsymbol{E}_{\mu}\right\}$ are orthonormal triads $\left\{\tilde{\boldsymbol{e}}_{\mu}\right\},\left\{\boldsymbol{e}_{\mu}\right\}$. For such a normalized normal basis $\left\{\boldsymbol{E}_{a}\right\}=\left\{\boldsymbol{n}, \tilde{\boldsymbol{e}}_{\mu}\right\}$, the components of $\tilde{h}_{a b}$ are $\tilde{h}_{\alpha \beta}=\tilde{\boldsymbol{e}}_{\alpha} \cdot \tilde{\boldsymbol{e}}_{\beta}=\delta_{\alpha \beta}$, and the metric components are

$$
g_{a b}=\operatorname{diag}(-1,+1,+1,+1) .
$$

One finds from (2.2)-(2.6) that

$$
\left[\boldsymbol{n}, \tilde{\boldsymbol{e}}_{\mu}\right]=\left(\varepsilon_{\mu}{ }^{\nu} \tilde{\Omega}^{\sigma}-\tilde{\theta}_{\mu}{ }^{v}\right) \tilde{\boldsymbol{e}}_{v},
$$

where $\tilde{\Omega}^{\sigma} \equiv \varepsilon^{\sigma \mu v} \tilde{e}_{\mu}{ }^{i} \tilde{e}_{v i ; j} n^{j}$ is the rate of rotation of the $\left\{\tilde{\boldsymbol{e}}_{\mu}\right\}$ along the normal congruence; the spatial commutators are given by (2.11). Using this basis one can write out the field Eqs. (2.8) and Jacobi identities (2.9) in tetrad form (in effect, one generalizes [1] to the case of an imperfect fluid (1.33)). One obtains the propagation equations

$$
\begin{gathered}
\partial_{0} a_{\alpha}+\varepsilon_{\alpha \beta \gamma} a^{\beta} \tilde{\Omega}^{\gamma}+\tilde{\sigma}_{\alpha \beta} a^{\beta}+\frac{1}{3} \tilde{\theta} a_{\alpha}=0, \\
\partial_{0} n^{\alpha \beta}+2 n_{\gamma}{ }^{(\alpha} \varepsilon^{\beta) \gamma \pi} \tilde{\Omega}_{\pi}-2 n_{\gamma}{ }^{\left({ }^{\alpha}\right.} \tilde{\sigma}^{\beta) \gamma}+\frac{1}{3} n^{\alpha \beta} \tilde{\theta}=0, \\
\partial_{0} \tilde{\theta}+\frac{1}{3} \tilde{\theta}^{2}+\tilde{\sigma}^{\alpha \beta} \tilde{\sigma}_{\alpha \beta}+\frac{1}{2} \mu\left(1+2 \sinh ^{2} \beta\right)+\frac{3}{2} p\left(1+\frac{2}{3} \sinh ^{2} \beta\right)=\Lambda, \\
\partial_{0} \tilde{\sigma}_{\beta \delta}=-\tilde{\theta} \tilde{\sigma}_{\beta \delta}+2 \tilde{\sigma}^{\kappa}{ }_{(\beta} \varepsilon_{\delta) \tau \kappa} \tilde{\Omega}^{\tau}+2 \varepsilon_{\tau \sigma \beta} n_{\delta)}{ }^{\tau} a^{\sigma}-2 n_{\delta \mu} n^{\mu}{ }_{\beta} \\
+n n_{\beta \delta}+\frac{1}{3} \delta_{\beta \delta}\left(2 n^{\kappa \tau} n_{\kappa \tau}-n^{2}\right) \\
+(\mu+p) \sinh ^{2} \beta\left(\tilde{c}_{\beta} \tilde{c}_{\delta}-\frac{1}{3} \delta_{\beta \delta}\right),
\end{gathered}
$$

(note that $\partial_{0} a_{\alpha}=d a_{\alpha} / d \tilde{t}$, etc.), and the constraint equations

$$
\begin{gathered}
3 a_{\kappa} \tilde{\sigma}_{\alpha}{ }_{\alpha}-\varepsilon_{\alpha \kappa \tau} \tau^{\tau \mu} \tilde{\sigma}^{\kappa}{ }_{\mu}-(\mu+p) \sinh \beta \cosh \beta \tilde{c}_{\alpha}=0, \\
-\frac{1}{3} \tilde{\theta}^{2}+\frac{1}{2} \tilde{\sigma}^{\alpha \beta} \tilde{\sigma}_{\alpha \beta}+3 a^{\alpha} a_{\alpha}+\frac{1}{2}\left(n^{\alpha \beta} n_{\alpha \beta}-\frac{1}{2} n^{2}\right)+\mu \cosh ^{2} \beta \\
+p \sinh ^{2} \beta+\Lambda=0 .
\end{gathered}
$$


Comparing (2.16c, d) for $\beta>0$ and $\beta=0$, one sees that the normals in a tilted model tend to contract faster than in the corresponding model without tilt, and that tilt tends to cause distortion of the normal congruence. Eq. (2.17a) determines the magnitude $(\mu+p) \sinh \beta \cosh \beta$ of $\tilde{q}_{a}$ by the relation

$$
(\mu+p) \sinh \beta \cosh \beta=3 \tilde{c}^{\alpha} \tilde{\sigma}_{\alpha \kappa} a^{\kappa}-\tilde{c}^{\alpha} \varepsilon_{\alpha \kappa} \eta^{\tau \mu} \tilde{\sigma}_{\kappa \mu},
$$

and determines the tilt direction $\tilde{c}_{\alpha}$ allowed for given $\tilde{\sigma}_{\alpha \beta}, a_{\alpha}$ and $n^{\alpha \beta}$. This relation shows that tilted models are only possible when the normals $n^{a}$ are distorting.

The tetrad form of the conservation Eqs. (1.34), (1.35) follow on noting that

$$
\begin{gathered}
\tilde{c}_{; a}^{a}=\tilde{c}_{a ; b} \tilde{h}^{a b}=-2 a_{\varepsilon} c^{\varepsilon}, \quad \tilde{h}^{\beta}{ }_{d}\left(\tilde{c}^{d}{ }_{; b} n^{b}\right)=\partial_{0} \tilde{c}^{\beta}+\varepsilon^{\beta \gamma \delta} \tilde{c}_{\gamma} \tilde{\Omega}_{\delta}, \\
\tilde{h}^{\delta}{ }_{e}\left(\tilde{c}_{; b}^{e} \tilde{c}^{b}\right)=\varepsilon_{\varepsilon \tau}^{\delta} \tilde{c}^{\delta} n^{\tau}{ }_{\beta} \tilde{c}^{\beta}+a^{\delta}-\tilde{c}^{\delta} a_{\beta} \tilde{c}^{\beta} .
\end{gathered}
$$

Further the tetrad components of the fluid expansion follow from (1.16) and (2.18a), and those of the vorticity and shear tensors from (1.26), (1.27) and

$$
\begin{aligned}
& \tilde{h}_{v}{ }^{a} \tilde{h}_{\mu}{ }^{b} \tilde{c}_{[a ; b]}=-\frac{1}{2} \tilde{\gamma}^{\kappa}{ }_{v \mu} \tilde{c}_{\kappa}=\varepsilon_{v \mu \sigma} \tilde{\omega}^{\sigma}, \quad \tilde{\omega}^{\sigma} \equiv-\frac{1}{2}\left(n^{\sigma \kappa}+\varepsilon^{\sigma \kappa \mu} a_{\mu}\right) \tilde{c}_{\kappa}, \\
& \tilde{h}_{v}{ }^{a} \tilde{h}_{\mu}{ }^{b} c_{(a ; b)}=n_{(v}{ }^{\tau} \varepsilon_{\mu) \sigma \tau} \tilde{c}^{\sigma}+a_{(v} \tilde{c}_{\mu)}-\delta_{v \mu} \tilde{c}^{\sigma} a_{\sigma}
\end{aligned}
$$

[by (1.21), these tensors are determined by their components perpendicular to $n^{a}$ ]. It follows that the components of the vorticity vector in the normal frame are

$$
\begin{gathered}
\omega^{0}=\frac{1}{2} \sinh ^{2} \beta \tilde{c}_{\alpha} n^{\alpha \beta} \tilde{c}_{\beta}, \\
\omega^{\alpha}=+\frac{1}{2} \tanh \beta\left(n^{\alpha \beta} \tilde{c}_{\beta}+\varepsilon^{\alpha \beta \gamma} \tilde{c}_{\beta} a_{\gamma}+\sinh ^{2} \beta \tilde{c}^{\alpha} \tilde{c}_{\beta} n^{\beta \gamma} \tilde{c}_{\gamma}\right) .
\end{gathered}
$$

Using these equations, one can check that as well as $\beta, a_{\alpha}, n^{\alpha \beta}$ and the eigenvalues of $n^{\alpha \beta}$, the quantities

$$
\tilde{c}_{\alpha} n^{\alpha \beta}, \tilde{c}_{\alpha} n^{\alpha \beta} \tilde{c}_{\beta}, \varepsilon^{\alpha \beta \gamma} a_{\beta} \tilde{c}_{\gamma}
$$

are I.R. ("invariant relation" quantities [5]): if they vanish or are non-zero at one time, the same is true at all times. This enables one to check (cf. [5]) that the group type and parameter value $h$ are preserved; further it shows that if $\tilde{c}_{\alpha}$ is a null eigenvector of $n^{\alpha \beta}$ at any time, the same is true at all times; and if it is parallel to $a_{\alpha}$, this is true at all times ${ }^{4}$. It follows from $(2.18 \mathrm{c})$ that $\omega^{a}$ is either zero or non-zero at all times (this is in fact simply a consequence of the existence of an effective equation of state $p=p(\mu)$, see $[7,8])$, and from the relation $\eta^{a b c d} \tilde{c}_{a} n_{b} \tilde{c}_{c ; d}=\tilde{c}_{\alpha} n^{\alpha \beta} \tilde{c}_{\beta}$ and (1.19) that if $\omega^{a}$ is perpendicular to $\tilde{c}^{a}$ at one time, this is true at all times.

${ }^{4}$ Note that $\varepsilon^{\alpha \beta \gamma} a_{\beta} \tilde{c}_{y}=0 \Rightarrow n^{\alpha \beta} \tilde{c}_{\beta}=0$, by (2.12). 
In fact the form of the propagation equation for $\tilde{q}_{\alpha} n^{\alpha \beta} \tilde{q}_{\beta}$ shows (cf. [5]) that (using (1.28))

$$
\begin{aligned}
& (w \cosh \beta)^{-3} \tilde{q}_{\alpha} n^{\alpha \beta} \tilde{q}_{\beta}=\text { constant } \Leftrightarrow \tilde{c}_{a} \omega^{a} \\
& =(\text { constant }) \times(\tanh \beta)^{-1} \cosh \beta \frac{w^{3}}{(\mu+p)^{2}},
\end{aligned}
$$

which is a conservation law for the component of $\omega^{a}$ parallel to the tilt direction.

One can also verify that, as must be the case, the constraint equations (2.17) are conserved as a consequence of the conservation equations (in fact the quantities on the left hand sides of (2.17) are I.R. quantities). Finally the way in which the vorticity components $\omega_{v \mu}$ are governed by the field Eqs. (2.17a) can be shown by combining this equation with $(2.18 \mathrm{c})$ and (1.26), obtaining

$$
(\mu+p) \cosh \beta \omega_{\alpha \beta}=\frac{1}{2}\left(\varepsilon_{\alpha \beta \sigma} n^{\sigma \kappa}+\delta_{\alpha}^{\kappa} a_{\beta}-\delta_{\beta}^{\kappa} a_{\alpha}\right)\left(\varepsilon_{\kappa \varrho \tau} n^{\tau v}-3 a_{\varrho} \delta_{\kappa}^{v}\right) \tilde{\sigma}_{v}^{\varrho},
$$

which determines $\omega_{\alpha \beta}$ algebraically from the distortion $\tilde{\sigma}_{v \mu}$ of the normal congruence.

To write out these equations in detail, one will usually specify $\left\{\tilde{\boldsymbol{e}}_{\mu}\right\}$ further. One way of doing this is to fit the triad $\left\{\tilde{\boldsymbol{e}}_{\mu}\right\}$ to the tensor $n^{\alpha \beta}$ and the vector $a_{\alpha}$ as in [1]: one chooses the $\left\{\tilde{\boldsymbol{e}}_{\mu}\right\}$ as an orthonormal triad such that

$$
n_{\alpha \beta}=\operatorname{diag}\left(n_{1}, n_{2}, n_{3}\right), \quad a_{\alpha}=(a, 0,0) .
$$

In a tilted universe, an alternative choice is given by defining the vectors $\boldsymbol{p}_{1}, \boldsymbol{p}_{2}$ as orthogonal unit vectors lying in the surfaces $S(t)$, and orthogonal to $\tilde{\boldsymbol{c}}$. They therefore span the 2-surfaces orthogonal to $\boldsymbol{n}$ and $\boldsymbol{c}$ at each point, and may equivalently be defined by the relations

$$
p_{a b}=p_{1 a} p_{1 b}+p_{2 a} p_{2 b}
$$

where the projection tensor $p_{a b}$ is defined by (1.30). In a tilted universe, these vectors are unique up to a rotation

$$
\boldsymbol{p}_{1}{ }^{\prime}=\boldsymbol{p}_{1} \cos \theta+\boldsymbol{p}_{2} \sin \theta, \quad \boldsymbol{p}_{2}{ }^{\prime}=\boldsymbol{p}_{2} \cos \theta-\boldsymbol{p}_{1} \sin \theta ;
$$

having partially used this freedom to ensure that $\boldsymbol{p}_{1}, \boldsymbol{p}_{2}$ are invariant under the group of isometries, the remaining freedom of choice is $(2.23 \mathrm{a})$ with

$$
\theta=\theta(t) \text {. }
$$

Using these vectors, a possible orthonormal basis $\left\{\tilde{\boldsymbol{e}}_{\mu}\right\}$ is given by the choice

$$
\left\{\tilde{\boldsymbol{e}}_{\mu}\right\}=\left\{\boldsymbol{p}_{1}, \boldsymbol{p}_{2}, \tilde{\boldsymbol{c}}\right\},
$$

unique precisely up to the rotation (2.23). 
For a normalized fluid basis $\left\{\boldsymbol{E}_{a}\right\}=\left\{r^{-1} \boldsymbol{u}, \boldsymbol{e}_{v}\right\}$, the components of $h_{a b}$ are $h_{\alpha \beta}=\boldsymbol{e}_{\alpha} \cdot \boldsymbol{e}_{\beta}=\delta_{\alpha \beta}$ and the metric tensor components are $g_{a b}$ $=\operatorname{diag}\left(-r^{-2},+1,+1,+1\right)$. One finds

$$
\left[r^{-1} \boldsymbol{u}, \boldsymbol{e}_{v}\right]=\gamma^{\mu}{ }_{0 v} \boldsymbol{e}_{\mu}=r^{-1}\left(\varepsilon^{\mu}{ }_{v \sigma} \Omega^{\sigma}-\theta^{\mu}{ }_{v}-\varepsilon^{\mu}{ }_{v \sigma} \omega^{\sigma}\right) \boldsymbol{e}_{\mu},
$$

where $\Omega^{\sigma} \equiv \varepsilon^{\sigma \mu v} e_{\mu}{ }^{i} e_{v i ; j} u^{j}$ is the rate of rotation of the $\left\{\boldsymbol{e}_{v}\right\}$ along the fluid congruence. The essential step in obtaining this result is to note that for any vector $\boldsymbol{e}$ orthogonal to $\boldsymbol{u}, \boldsymbol{u} \cdot\left[r^{-1} \boldsymbol{u}, \boldsymbol{e}\right]=-r^{-2} e^{a}\left(r_{, a}+r \dot{u}_{a}\right)$; and this vanishes by (1.22), (1.23). Further

$$
\left[\boldsymbol{e}_{\mu}, \boldsymbol{e}_{\nu}\right]=-2 r \varepsilon_{\mu \nu \sigma} \omega^{\sigma}\left(u r^{-1}\right)+\gamma^{\kappa}{ }_{\mu \nu} \boldsymbol{e}_{\kappa} .
$$

To specify the basis further, an obvious choice is to use (2.22), defining

$$
\left\{\boldsymbol{e}_{v}\right\}=\left\{\boldsymbol{p}_{1}, \boldsymbol{p}_{2}, \boldsymbol{c}\right\},
$$

with the freedom of choice (2.23). The commutation coefficients (2.25), (2.26) for this basis can easily be related to those for the basis (2.24) by using the relations (1.12) between $\boldsymbol{c}$ and $\tilde{c}$. Useful identities for this basis are obtained by applying the relations

$$
\partial_{a}\left(\partial_{b} f\right)-\partial_{b}\left(\partial_{a} f\right)=\gamma_{a b}^{c} \partial_{c} f
$$

which [by (2.3), (2.4)] hold for any function $f$, to the time coordinate $t$ defined by (2.13); for $a=0, b=v$ one finds

$$
\gamma_{01}^{3}=\gamma_{02}^{3}=0, \gamma_{03}^{3}=d \log (r \tanh \beta) / d t
$$

[which is really a way of writing (1.29)], and for $a=\mu, b=v$

$$
2 \varepsilon_{\mu v \sigma} \omega^{\sigma}=\gamma_{\mu \nu}^{3} \tanh \beta
$$

[which is essentially a way of writing (1.26)]. These relations are the integrability conditions ensuring that the choice of tetrad and the choice of coordinate $t$ are compatible. To simplify the rotation coefficients further, one can use the freedom of choice (2.23) of $\left\{\boldsymbol{p}_{v}\right\}$ to set

$$
\gamma_{01}^{2}=0
$$

which leaves freedom (2.23a) with $\theta$ a constant. The field Eqs. (2.8) and Jacobi identities (2.9) with this tetrad choice, i.e. with (2.25)-(2.31) holding, are written out explicitly in Appendix A. It is sometimes convenient to use the renormalized tilt parameter

$$
\lambda \equiv r \tanh \beta
$$

in later work [cf. Eq. (2.29)], and this abbreviation is used in the Appendix. In Appendix B we give the quantities $n_{\alpha \beta}$ and $a_{\varepsilon}$ in terms of the fluid $\gamma_{b c}^{a}$. The conservation Eqs. (1.22) now imply

$$
\lambda^{\prime} / \lambda=r \lambda, o / \lambda=-\theta_{3} .
$$


Defining $l_{(3)}$ by $l_{(3)} ; / l_{(3)}=\theta_{3}$ this equation has the integrated form ${ }^{5}$

$$
\lambda=(\text { constant }) \times l_{(3)}{ }^{-1} .
$$

Using (2.33), one can show that four of the Jacobi identities $\left(\begin{array}{c}3 \\ a b c\end{array}\right)$ in the notation of [12]) are equivalent to four other Jacobi identities $\left(\left(\begin{array}{c}0 \\ a b c\end{array}\right)\right.$ in [12]). This fact has been used in writing out the equations in Appendix A.

\section{Particular Group Types}

One can now take the "normal" field equations and identities (2.16), (2.17), and (1.34), and use them to examine properties of universe models invariant under any particular group. A convenient choice of basis for explicitly writing out these equations is a normalized normal basis such that (2.21) holds; then the form of the equations is precisely that of Eqs. (2.11), (2.12), and (3.2)-(3.5) in Appendix I of [1], except that the right hand side of each $(0 v)$ equation is replaced by a term $+\tilde{q}_{v}$, the right hand side of each $(\mu v)$ equation has a term $-\tilde{\pi}_{\mu v}$ added to it, and the quantities $\theta, \theta_{v}, \sigma_{\mu v}, \Omega_{v}, \mu$ and $p$ each have a $\sim$ sign added to them.

Class $A$ universes are those in which $a_{\varepsilon}=0 \Leftrightarrow a=0$. Then by (2.18a), $\tilde{c}_{a: b} \tilde{h}^{a b}=0$, so one can immediately write down an integral of (1.28) (cf. [6]) and then rewrite (2.19):

$$
w \tilde{l}^{3} \cosh \beta=\text { constant }, \quad\left(\tilde{q}_{\alpha} n^{\alpha \beta} \tilde{q}_{\beta}\right) \tilde{l}^{9}=\text { constant } .
$$

Using a normalized normal basis, the vorticity components $\omega_{v \mu}$ are

$$
\omega_{v \mu}=-\frac{1}{2} \sinh \beta \varepsilon_{v \mu \sigma} n^{\sigma \kappa} \tilde{c}_{\kappa}
$$

when $a_{\varepsilon}=0$; it follows that vanishing of vorticity corresponds to vanishing of the I.R. quantity $n^{\sigma \kappa} \tilde{c}_{\kappa}$.

Theorem 3.1. There are no tilted models of type I; tilted models of type II have zero vorticity. Tilted models of types VIII or IX have nonzero vorticity.

For a type I model universe, $n^{\alpha \beta}=a_{\varepsilon}=0$, so (2.17a) shows $\beta=0$ (cf. [11]), i.e. there are no tilted solutions. In a type II universe, using a basis (2.21) with $n_{2}=n_{3}=0$, the (01) equation shows $\beta \tilde{c}_{1}=0$; then (3.2) shows $\omega_{v \mu}=0$, which implies $\omega_{a b}=0$ (this generalizes to a perfect fluid Oszvath's result [6] for dust). In a universe of type VIII or IX, det $n^{\alpha \beta} \neq 0$, so for any $\tilde{c}_{\alpha}, n^{\alpha \beta} \tilde{c}_{\alpha} \neq 0$, and hence $\beta \neq 0 \Rightarrow \omega_{v \mu} \neq 0$ by (3.2).

${ }^{5}$ Eqs. (2.25) and (2.34) are really integrated forms of the conservation equations. Here we have essentially used the method of integrating these equations due to Taub [34], and used by him and MacCallum in investigating spatially homogeneous cosmological models [35]. 
Class $B$ universes are those in which $a_{\beta} \neq 0 \Leftrightarrow a \neq 0$; using the basis (2.21), $n_{1}=0$ because of the identity (2.12). The vorticity vanishes if and only if the quantity $\tilde{\omega}^{\sigma}$ defined by $(2.18 \mathrm{c})$ vanishes; in this frame,

$$
2 \tilde{\omega}^{1}=-n_{1} \tilde{c}_{1}=0,2 \tilde{\omega}^{2}=-n_{2} \tilde{c}_{2}+a \tilde{c}_{3}, 2 \tilde{\omega}^{3}=-a \tilde{c}_{2}-n_{3} \tilde{c}_{3} .
$$

Hence $\omega^{a}=0$ if $\tilde{c}_{2}=\tilde{c}_{3}=0$, and also in particular cases when at least one of $\tilde{c}_{2}, \tilde{c}_{3}$ is non-zero and the determinant $n_{2} n_{3}+a^{2}$ vanishes; this means that the group is Bianchi type III (it is type $\mathrm{VI}_{h}$ with $h=-1$ ). The case $\tilde{c}_{2}=\tilde{c}_{3}=0$ is the case when the I.R. variable $\varepsilon^{\alpha \beta \gamma} \tilde{c}_{\beta} a_{\gamma}$ vanishes. Then the (02), (03) equations show that either $\tilde{\sigma}_{12}=\tilde{\sigma}_{13}=0$, or the determinant $n_{2} n_{3}+9 a^{2}$ vanishes; this means the group is type $\mathrm{VI}_{h}$ with $h=-1 / 9$ (this case is a straightforward generalization of class Bbii of [1]).

Theorem 3.2. If $\tilde{c}_{\alpha}$ is parallel to $a_{\alpha}$ in a tilted class $B$ universe, then $\omega^{a}=0$, and either these vectors are both eigenvectors of the normal shear $\tilde{\sigma}_{a b}$, or the group is type $V I_{h}$ with $h=-1 / 9$. If $\tilde{c}_{\alpha}$ is not parallel to $\tilde{a}_{\alpha}$, then $\omega^{a} \neq 0$ except in particular Bianchi III solutions.

(In fact examination of the conservation equations shows that in class B universes, the effect of the $\boldsymbol{a}$-vector is to tend to swing the tilt direction $\boldsymbol{c}$ towards the $-\boldsymbol{a}$-direction.)

When $\omega^{a} \neq 0$, one can obtain more detailed information by considering the I.R. quantity $\tilde{c}_{\alpha} n^{\alpha \beta} \tilde{c}_{\beta}$, as $\tilde{c}_{a} \omega^{a}$ and $n_{a} \omega^{a}$ vanish if and only if this quantity does. Using the basis (2.21), it takes the form

$$
\tilde{c}_{\alpha} n^{\alpha \beta} \tilde{c}_{\beta}=\left(\tilde{c}_{1}\right)^{2} n_{1}+\left(\tilde{c}_{2}\right)^{2} n_{2}+\left(\tilde{c}_{3}\right)^{2} n_{3} .
$$

This clearly vanishes in a type $\mathrm{V}$ universe; however it must be non-zero in models of type $\mathrm{VII}_{0}, \mathrm{VII}_{h}$ and IX when $\omega^{a} \neq 0$ (in these cases, the non-vanishing $n_{v}$ are all positive).

Theorem 3.3. In rotating universe of type $V I I_{0}, V I I_{h}$ and $I X$, the vorticity direction cannot be perpendicular to the tilt direction (i.e. $\left.\omega^{a} \tilde{c}_{a} \neq 0\right)$; but in a tilted universe of type $V$, these vectors necessarily are perpendicular (i.e. $\omega_{a} \tilde{c}^{a}=0$ ). When $\omega^{a} \tilde{c}_{a} \neq 0,(2.19)$ gives a conservation law for this quantity.

Thus apart from type $\mathrm{V}$, one can have rotating universes with $\omega^{a}$ perpendicular to $\tilde{c}^{a}$ only in special cases of types $\mathrm{VI}_{0}$, VIII, IV and $\mathrm{VI}_{h}$. The simplest such universes are those in which the I.R. variable $n^{\alpha \beta} \tilde{c}_{\beta}$ vanishes. This cannot happen in rotating type $\mathrm{VI}_{h}$ universes (for vanishing $n^{\alpha \beta} \tilde{c}_{\beta}$ would mean that $\tilde{c}_{\alpha}$ was parallel to $a_{\alpha}$ ). 
Theorem 3.4. The rotating tilted models in which $n^{\alpha \beta} \tilde{c}_{\beta}$ vanish are all type $V$ rotating universes, and particular type $I V$ rotating universes. In these spaces,

$$
\omega^{a}=\frac{1}{2} \tanh \beta \eta^{a b c d} n_{b} a_{c} \tilde{c}_{d},
$$

so $\omega^{a}$ is perpendicular to each of $n^{a}, a^{b}$ and $\tilde{c}^{d}$.

One can also consider the possibility of rotating universes having $\omega^{a}$ parallel to $c^{a}$ (i.e. lying in the plane of $\tilde{c}^{a}$ and $n^{a}$ ). In this case, $(2.18 \mathrm{e}$ ) shows that

$$
n^{\alpha \beta} \tilde{c}_{\beta}+\varepsilon^{\alpha \beta \gamma} \tilde{c}_{\beta} a_{\gamma}=\tilde{c}^{\alpha}\left(\tilde{c}_{\beta} n^{\beta \gamma} \tilde{c}_{\gamma}\right) \neq 0 \Rightarrow a_{\beta} \tilde{c}^{\beta}=0,
$$

where $\tilde{c}_{\alpha} h^{\alpha \beta} \tilde{c}_{\beta}$ cannot vanish because if $\omega^{a}$ is non-zero and parallel to $c^{a}$, then $\omega^{a} \tilde{c}_{a}$ cannot vanish; the implication follows on multiplying the first equation by $a_{\alpha}$. It follows from (3.5) that in class $A$ universes, $\omega^{a}$ is parallel to $c^{a}$ if and only if $\tilde{c}_{a}$ is an eigenvector of $n^{\alpha \beta}$. Choosing a basis (2.21) with $\tilde{c}_{\alpha}=(1,0,0)$, then $\omega^{a} \neq 0 \Rightarrow n_{1} \neq 0$, and the conservation equations and Jacobi identities show $\tilde{\Omega}_{3}=\tilde{\sigma}_{12}=0, \tilde{\Omega}_{2}=-\tilde{\sigma}_{13}=0$. These conditions are then consistent with the other field equations; use of (1.27) and (2.18) shows that $\omega^{a}$ is an eigenvector of the shear $\sigma_{a b}$ and (1.32) shows that it is Fermi propagated along the fluid flow lines.

In class B universe, (3.5) implies that $\tilde{c}_{\alpha}$ must be perpendicular to $a_{\alpha}$. To proceed further, it is convenient to use the normalised fluid basis of $\S 2$ and the equations in Appendix A, instead of the normal basis used so far in this section. In the new basis, $c^{1}=c^{2}=0, c^{3} \neq 0$, so $\omega^{1}=\omega^{2}=0$, $\omega^{3} \neq 0$; the Jacobi identities show $\sigma_{13}=\sigma_{23}=0$. The condition $\tilde{c}_{\alpha} a^{\alpha}=0$ shows $a^{3}=0$; then at least one of $\gamma_{12}^{2}$ or $\gamma^{1}{ }_{12}$ is non-zero (or $a_{\alpha}$ would be zero). By the (23) and (13) equations, this is only possible if $\gamma_{13}^{1}=\gamma^{2}{ }_{23}$ and $\gamma_{23}^{1}+\gamma_{13}^{2}=0$, and by the (01), (02) equations, it is only possible if $\theta_{11}=\theta_{22}, \sigma_{12}=0$. The conditions we now have are sufficient to show that the spacetime is locally rotationally symmetric, or L.R.S. (see [12, 13] and $\S 4$ below); but there are no such rotating solutions (see $\S 4$ ). Hence there are no rotating class B solutions with $\omega^{a} \| c^{a}$.

Theorem 3.5. The rotating tilted models with $\omega^{a}$ parallel to $c^{a}$ are the rotating class $A$ solutions with $c_{\alpha}$ an eigenvector of $n^{\alpha \beta}$. In these universes $\omega^{a}$ and $c^{a}$ are Fermi propagated eigenvectors of the shear $\sigma_{a b}$, while $\tilde{c}_{a}$ is an eigenvector of the normal shear $\tilde{\sigma}_{a b}$; further $\omega_{a} c^{a} \neq 0$.

The two simplest group types allowing tilted universe models are types II and V. In each of these cases one can obtain simple geometrically characterized tetrads and write down associated simple coordinate systems. The type $\mathrm{V}$ case can in fact be easily ex tended to cover all type IV models in which $n^{\alpha \beta} \tilde{c}_{\beta}=0$ (cf. Theorem 3.4).

In the case of a type II universe, one can use a basis (2.21) with $n_{1} \neq 0$, $n_{2}=n_{3}=a=0 ;$ a rotation in the $\left(\tilde{\boldsymbol{e}}_{2}, \tilde{\boldsymbol{e}}_{3}\right)$ plane can be used to set $\tilde{c}_{2}=0$, and then (assuming $\beta>0$ ) the tetrad is unique. The Jacobi identities and 
the (01), (02) and (13) equations show that $\tilde{c}_{1}=0, \tilde{\sigma}_{13}=\tilde{\Omega}_{2}=0, \tilde{\Omega}_{3}=-\tilde{\sigma}_{12}$, $\tilde{\Omega}_{1}=-\tilde{\sigma}_{23}$. Coordinates can be chosen so that the tetrad-coordinate relations $\tilde{\boldsymbol{e}}_{a}=\tilde{e}_{a}^{i} \partial / \partial x^{i}$ take the form ${ }^{6}$

$$
\begin{gathered}
\tilde{\boldsymbol{e}}_{0}=\partial / \partial t, \tilde{\boldsymbol{e}}_{1}=X(t)^{-1} \partial / \partial x^{1}, \quad \tilde{\boldsymbol{e}}_{2}=Y(t)^{-1}\left(\partial / \partial x^{2}+f\left(t, x^{3}\right) \partial / \partial x^{1}\right), \\
\tilde{\boldsymbol{e}}_{3}=Z(t)^{-1}\left(\partial / \partial x^{3}+g(t) \partial / \partial x^{2}+h\left(t, x^{3}\right) \partial / \partial x^{1}\right)
\end{gathered}
$$

where $h=\int f d g / d t d t$; the metric components $g_{i j}$ can now be easily found by first obtaining the inverse matrix $\tilde{e}^{a}{ }_{i}$ and then using (2.1b), (2.14). The Jacobi identities and (12), (23) field equations show

$$
n_{1}=N_{1} X(Y Z)^{-1}, \quad \tilde{\sigma}_{12}=\Sigma_{12}\left(X^{2} Z\right)^{-1}, \quad \tilde{\sigma}_{23}=\Sigma_{23}\left(Y^{2} X\right)^{-1},
$$

where $N_{1}, \Sigma_{12}, \Sigma_{23}$ are constants, which shows that the functions $f, g$ are determined by

$$
f=-N_{1} x^{3}-2 \Sigma_{12} \int Y\left(X^{3} Z\right)^{-1} d t, \quad g=-2 \Sigma_{23} \int Z\left(X Y^{3}\right) d t .
$$

The conservation Eqs. (1.28), (1.31) can be integrated in the form $w X Y Z \cosh \beta=W_{0}, r Z \sinh \beta=R_{0}, W_{0}>0$ and $R_{0}$ constants.

The remaining field equations consist of the time development equations

$$
\begin{aligned}
& \frac{X^{*}}{X}+\frac{X^{\cdot}}{X} \frac{Y^{\cdot}}{Y}+\frac{X^{\cdot}}{X} \frac{Z^{\cdot}}{Z}+\frac{1}{2}\left(N_{1}\right)^{2} \frac{X^{2}}{Y^{2} Z^{2}} \\
&-2 \frac{\left(\Sigma_{12}\right)^{2}}{X^{4} Z^{2}}=\frac{1}{2}(\mu-p)+\Lambda, \\
& \frac{Y^{*}}{Y}+\frac{Y^{\cdot}}{Y} \frac{Z}{Z}+\frac{Y^{\cdot}}{Y} \frac{X^{\cdot}}{X}-\frac{1}{2}\left(N_{1}\right)^{2} \frac{X^{2}}{Y^{2} Z^{2}} \\
& \quad-2 \frac{\left(\Sigma_{23}\right)^{2}}{Y^{4} X^{2}}+2 \frac{\left(\Sigma_{12}\right)^{2}}{X^{4} Z^{2}}=\frac{1}{2}(\mu-p)+\Lambda, \\
& \frac{Z^{\cdot \cdot}}{Z}+\frac{Z}{Z} \frac{X^{\cdot}}{X}+\frac{Z^{\cdot}}{Z} \frac{Y^{\cdot}}{Y}-\frac{1}{2}\left(N_{1}\right)^{2} \frac{X^{2}}{Y^{2} Z^{2}} \\
&+2 \frac{\left(\Sigma_{23}\right)^{2}}{Y^{4} X^{2}}=\frac{1}{2}(\mu-p)+\sinh ^{2} \beta(\mu+p)+\Lambda,
\end{aligned}
$$

and the two first integrals

$$
\begin{gathered}
(\mu+p) X Y Z^{2} \sinh \beta \cosh \beta=\Sigma_{12} N_{1} \Leftrightarrow R_{0} W_{0}=\Sigma_{12} N_{1}, \\
\frac{X^{\cdot}}{X} \frac{Y^{\cdot}}{Y}+\frac{Y^{\cdot}}{Y} \frac{Z}{Z}+\frac{Z}{Z} \frac{X^{\cdot}}{X}=\frac{1}{4}\left(N_{1}\right)^{2} \frac{X^{2}}{Y^{2} Z^{2}} \\
+\frac{\left(\Sigma_{12}\right)^{2}}{X^{4} Z^{2}}+\frac{\left(\Sigma_{23}\right)^{2}}{Y^{4} X^{2}}+\mu \cosh ^{2} \beta+p \sinh ^{2} \beta+\Lambda .
\end{gathered}
$$

${ }^{6}$ The time coordinate $t$ in (3.6), (3.11), (4.8) and (6.4) is in fact the special coordinate $\tilde{t}$ defined by (1.4). 
The fluid velocity is given by $\boldsymbol{u}=\cosh \beta \tilde{\boldsymbol{e}}_{0}+\sinh \beta \tilde{\boldsymbol{e}}_{3}$. The constants $W_{0}$, $R_{0}, \Sigma_{12}, N_{1}$, and $\Sigma_{23}$ all represent intrinsic properties of the solution; in a tilted universe, $\beta>0 \Rightarrow R_{0} \Sigma_{12} N_{1} \neq 0$. Given an equation of state $p=p(\mu),(3.8)$ determines $p, \mu$ and $\beta$ in terms of $X, Y, Z$, and then (3.10) and one of (3.9) will determine $X, Y, Z$ and so $f, g$ and $h$. The simplest class of solutions is that in which $\Sigma_{23}=0$; this is the case in which $c^{a}$ is an eigenvector of the shear tensor $\sigma_{a b}$.

In a type $V$ universe or a type IV universe with $n^{\alpha \beta} \tilde{c}_{\beta}=0$, one can always find a basis $(2.21)$ with $n_{1}=n_{3}=0, \tilde{c}_{\alpha}=(\cos \theta, 0, \sin \theta)$. The Jacobi identities and (02), (13) equations show $\tilde{\Omega}_{3}=\tilde{\sigma}_{12}=0, \tilde{\Omega}_{2}=-\tilde{\sigma}_{13}$; and that either $\tilde{\Omega}_{1}=-\tilde{\sigma}_{23}$, or the basis can be chosen so that this relation is fulfilled. Coordinates can be chosen so that the tetrad-coordinate relations $\tilde{\boldsymbol{e}}_{a}=\tilde{e}_{a}{ }^{i} \partial / \partial x^{i}$ take the form

$$
\begin{gathered}
\tilde{\boldsymbol{e}}_{0}=\partial / \partial t, \quad \tilde{\boldsymbol{e}}_{1}=X^{-1}(t)\left(\partial / \partial x^{1}+f\left(t, x^{1}, x^{3}\right) \partial / \partial x^{2}+g\left(t, x^{1}\right) \partial / \partial x^{3}\right) \\
\tilde{\boldsymbol{e}}_{2}=Y^{-1}(t) \exp \left(A_{0} x^{1}\right) \partial / \partial x^{2} \\
\tilde{\boldsymbol{e}}_{3}=Z^{-1}(t) \exp \left(A_{0} x^{1}\right)\left(\partial / \partial x^{3}+k(t) \partial / \partial x^{2}\right)
\end{gathered}
$$

which determines the metric tensor components. The Jacobi identities and (13), (23) field equations show that

$$
\begin{gathered}
a=A_{0} X^{-1}, \quad n_{2}=N_{2} Y(X Z)^{-1}, \\
\tilde{\sigma}_{13}=\Sigma_{13}(t)\left(Y Z^{2}\right)^{-1}, \quad \tilde{\sigma}_{23}=\Sigma_{23}(t)\left(X Y^{2}\right)^{-1},
\end{gathered}
$$

where $A_{0}, N_{2}$ are constants and

$$
\partial_{0} \Sigma_{13}=Y Z^{2}(\mu+p) \sinh ^{2} \beta \sin \theta \cos \theta, \partial_{0} \Sigma_{23}=-A_{0} N_{2} Y^{3}(X Z)^{-1} ;
$$

and the functions $f, g, k$ are given by

$$
\begin{gathered}
k=-2 \int \Sigma_{23} Z\left(X Y^{3}\right)^{-1} d t, f=N_{2} x^{3}-2 \exp \left(A_{0} x^{1}\right) \int k \Sigma_{13} X\left(Y Z^{3}\right)^{-1} d t \\
g=-2 \exp \left(A_{0} x^{1}\right) \int \Sigma_{13} X\left(Y^{2} Z^{2}\right)^{-1} d t
\end{gathered}
$$

The conservation equations can be written in the form

$$
\begin{gathered}
d(\log w X Y Z \cosh \beta) / d t=2 \tanh \beta \cos \theta A_{0} X^{-1} \\
d \log (r \sinh \beta) / d t \\
=-\left(X^{\cdot} X^{-1} \cos ^{2} \theta+2 \sin \theta \cos \theta \Sigma_{13}\left(Y Z^{2}\right)^{-1}+Z Z^{-1} \sin ^{2} \theta\right) \\
d\left((\mu+p) \sinh \beta \cosh \beta \sin \theta X Y Z^{2}\right) / d t \\
\quad-Y Z^{2}(\mu+p) \sinh ^{2} \beta 3 A_{0} \sin \theta \cos \theta=0
\end{gathered}
$$


The remaining field equations consist of the time development equations

$$
\begin{aligned}
\frac{X^{*}}{X}+\frac{X^{\cdot}}{X} \frac{Y^{\cdot}}{Y}+ & \frac{X^{\cdot}}{X} \frac{Z}{Z}-2 \frac{A_{0}^{2}}{X^{2}}-\frac{1}{2} \frac{N_{2}^{2} Y^{2}}{X^{2} Z^{2}}+2 \frac{\Sigma_{13}^{2}}{Y^{2} Z^{4}} \\
& =\frac{1}{2}(\mu-p)+(\mu+p) \sinh ^{2} \beta \cos ^{2} \theta+\Lambda, \\
\frac{Y^{*}}{Y}+\frac{Z}{Z} \frac{Y^{\cdot}}{Y}+ & \frac{Y^{\cdot}}{Y} \frac{X^{\cdot}}{X}-2 \frac{A_{0}^{2}}{X^{2}}+\frac{1}{2} \frac{N_{2}^{2} Y^{2}}{X^{2} Z^{2}}-2 \frac{\Sigma_{23}^{2}}{X^{2} Y^{4}} \\
& =\frac{1}{2}(\mu-p)+\Lambda, \\
\frac{Z^{*}}{Z}+\frac{Z}{Z} \frac{X^{\cdot}}{X}+ & \frac{Z^{\cdot}}{Z} \frac{Y^{\cdot}}{Y}-2 \frac{A_{0}^{2}}{X^{2}}-\frac{1}{2} \frac{N_{2}^{2} Y^{2}}{X^{2} Z^{2}}-2 \frac{\Sigma_{13}^{2}}{Y^{2} Z^{4}} \\
+ & 2 \frac{\Sigma_{23}^{2}}{X^{2} Y^{4}}=\frac{1}{2}(\mu-p)+(\mu+p) \sinh ^{2} \beta \sin ^{2} \theta+\Lambda,
\end{aligned}
$$

and the three first integrals

$$
\begin{aligned}
& \frac{A_{0}}{X}\left(2 \frac{X^{\cdot}}{X}-\frac{Y^{\cdot}}{Y}-\frac{Z^{*}}{Z}\right)+\frac{\Sigma_{23} N_{2}}{X^{2} Y Z}=(\mu+p) \sinh \beta \cosh \beta \cos \theta, \\
& 3 \frac{A_{0}}{X} \frac{\Sigma_{13}}{Y Z^{2}}=(\mu+p) \sinh \beta \cosh \beta \sin \theta, \\
& \frac{X^{\cdot}}{X} \frac{Y^{\cdot}}{Y}+\frac{Y^{\cdot}}{Y} \frac{Z^{\cdot}}{Z}+\frac{Z}{Z} \frac{X^{\cdot}}{X}=3 \frac{A_{0}^{2}}{X^{2}}+\frac{1}{4} \frac{N_{2}^{2} Y^{2}}{X^{2} Z^{2}}+\frac{\Sigma_{13}^{2}}{Y^{2} Z^{4}} \\
& +\frac{\Sigma_{23}^{2}}{X^{2} Y^{4}}+\mu \cosh ^{2} \beta+p \sinh ^{2} \beta+\Lambda .
\end{aligned}
$$

The fluid 4-velocity is given by $\boldsymbol{u}=\cosh \beta \tilde{\boldsymbol{e}}_{0}+\sinh \beta\left(\cos \theta \tilde{\boldsymbol{e}}_{1}+\sin \theta \tilde{\boldsymbol{e}}_{3}\right)$. In general this set of equations for $p, \mu, \beta, \theta ; \Sigma_{13}, \Sigma_{23} ; X, Y, Z$ is rather complex; given an equation of state $p(\mu)$, one can take the conservation equations as determining $p, \mu, \beta, \theta$; Eqs. (3.12) determine the $\Sigma_{i j}$; and the first integrals (3.16) determine $X, Y, Z$. The vorticity vanishes if and only if $\Sigma_{13}=0 \Leftrightarrow \sin \theta=0 \Leftrightarrow \tilde{q}_{3}=0$. The type IV solutions have $N_{2} \neq 0$ which implies $\Sigma_{23} \neq 0$; the tetrad is unique. The type V solutions have $N_{2}=0$; the tetrad is unique if the vorticity is non-zero; $\Sigma_{23}$ is constant, and the simplest such rotating solutions are those in which $\Sigma_{23}=0$. These are the type $\mathrm{V}$ rotating solutions in which $\omega^{a}$ is an eigenvector of the shear $\sigma_{a b}$. In the non-rotating type $V$ solutions, one can set $\Sigma_{z_{3}}=0$ by a rotation of the $\left(\tilde{\boldsymbol{e}}_{2}, \tilde{\boldsymbol{e}}_{3}\right)$ vectors; the tetrad is then unique if $\tilde{\theta}_{2} \neq \theta_{3}$, and the equations are hardly more complicated than in the type II solutions discussed above. Finally the simplest solution of all is the special non-rotating type $V$ case in which $\tilde{\theta}_{2}=\tilde{\theta}_{3}, \Sigma_{23}=0$; in this case one can set $Y=Z$, and one has an L.R.S. solution (see $\S 4$ ) which can be completely integrated for simple 
equations of state. One has only to solve 2 conservation equations for $p, \mu, \beta$, together with two first integral equations for $X, Y$.

It is apparent, on comparing with [1], that the equations in the tilted (and particularly, the rotating) universes are rather more complex than in the non-tilted case. Exact properties of tilted models of type IX have been given in $[5,6,11,14]$; properties of class A solutions with $c_{\alpha}$ an eigenvector of $n^{\alpha \beta}$ in $[14 ; 6]$; of non-rotating type $\mathrm{V}$ solutions in [5]; and of $\mathrm{VII}_{0}$ and $\mathrm{VII}_{h}$ solutions in [15]. Exact tilted solutions have been given in [12], by Farnsworth in [16] (a non-rotating L.R.S. solution), and by Demianski and Grischuk in [17] (a rotating and expanding solution); these will be discussed in the next section. Dynamic properties of tilted universes except type IX have been examined by Collins and Hawking [15]; type V by Hawking [18], Matzner [19] and others $([24,25])$; and of type IX by a number of authors $([18,20-23])$.

\section{Tilted Models with Higher Symmetry}

There are various kinds of further symmetry conditions one can impose on tilted homogeneous cosmological models; we shall examine two of these in this section.

First we consider models which are L.R.S. (locally rotationally symmetric: see $[12,13])$. Then there is a group of rotations at each point such that all covariantly defined quantities are invariant under it. As $\boldsymbol{u}, \boldsymbol{n}, \boldsymbol{c}, \tilde{\boldsymbol{c}}$ are uniquely defined in any tilted model, in an L.R.S. tilted model the rotation must take place in the 2-plane orthogonal to $\boldsymbol{u}, \boldsymbol{n}, \boldsymbol{c}, \tilde{\boldsymbol{c}}$ spanned by $p_{a b}$; so all covariantly defined quantities are invariant under the rotation (2.23a). Choose a normalized normal basis with triad (2.24); then it follows $([1 ; 12,13])$ that

$$
\tilde{\theta}_{1}=\tilde{\theta}_{2}, \tilde{\sigma}_{\mu \nu}=0(\mu \neq v), \tilde{\Omega}_{\alpha}=(0,0, \tilde{\Omega}), \tilde{c}_{\varepsilon}=(0,0,1),
$$

and either

or

$$
\begin{aligned}
& n_{\mu \nu}=\operatorname{diag}(n, n, m), \quad a_{\varepsilon}=(0,0, a), \\
& n_{\mu \nu}=0 \operatorname{except} n_{23}=n_{32}=a, \quad a_{\varepsilon}=(a, 0,0) .
\end{aligned}
$$

In the second case one can, after adding suitable terms in $\tilde{q}_{v}$ and $\tilde{\pi}_{\mu \nu}$, use the equations of Appendix II of [1], with $r=0$. By the (03) equation, $\tilde{\sigma}_{13}=0 \Rightarrow \tilde{q}_{3}=0$; however by (4.1) the $\tilde{q}_{v}$ vector must lie in the 3 -direction, so there can be no such tilted models. In the first case, when (4.2a) holds, one can use the equations of Appendix I of [1] as before, after suitable renumbering. We therefore renumber, and conditions (4.1), (4.2a) become

$$
\begin{gathered}
\tilde{\theta}_{1} \equiv \alpha, \tilde{\theta}_{2}=\tilde{\theta}_{3} \equiv \gamma, \tilde{\sigma}_{\mu \nu}=0(\mu \neq v), \tilde{\Omega}_{\varepsilon}=(\tilde{\Omega}, 0,0), \tilde{c}_{\varepsilon}=(1,0,0), \\
n_{\mu \nu}=\operatorname{diag}(m, n, n), a_{v}=(a, 0,0) .
\end{gathered}
$$


In the class A solutions, $a=0$; but then the $(0 v)$ equations immediately imply $\tilde{q}_{v}=0$, so there are no such tilted L.R.S. models. In the class B solutions, $a \neq 0, m=0$; now (3.3) shows that $\tilde{\omega}^{\varepsilon}=0$, so there are no rotating L.R.S. tilted homogeneous universe models.

An examination of the field equations shows that neither $\tilde{\Omega}$ nor $n$ play any part in the remaining non-trivial field equations, which are two time derivative equations and two first integrals. This indicates that these quantities are non-essential; and in fact $\tilde{\Omega}$ can be set to zero by a rotation in the $\left(\boldsymbol{e}_{2}, \boldsymbol{e}_{3}\right)$ plane which varies with time, and $n$ can be set to zero by such a rotation which varies spatially (this is just the transformation between a type $\mathrm{V}$ and type $\mathrm{VII}_{h}$ basis in an L.R.S. space, which was examined in [1]).

Theorem 4.1. The tilted L.R.S. models are invariant under a group of type $V$, and a group of type $V I I_{h}$ for each value of $h>0$. The fluid vorticity vanishes in these universes.

Using a basis (4.3) in which $\tilde{\Omega}=m=n=0$, the field equations now only involve $\mu, p, \beta ; \alpha, \gamma$ and $a$. As the universe is type $\mathrm{V}$, one can choose coordinates as in $\S 3$; then the metric is that given by (3.11), with $f=g$ $=k=0, Y(t)=Z(t)$. As there is no rotation, one has $\sin \theta=0, \Sigma_{13}=0$ $=\Sigma_{23}$; the conservation equations are given by the first two of (3.14) (with $\cos \theta=1$ ), and the remaining field equations can be taken to be the two first integrals (3.16) with $N_{2}=0$. The solution in the case of vanishing pressure has been obtained by Farnsworth ([16]; see also [12]). When $p \neq 0$ one can for particular equations of state reduce the equations to one second order equation and a set of quadratures, but solution of the equations seems difficult. It seems clear that one has more likelihood of obtaining solutions by changing to a fluid tetrad and associated coordinates [one obtains such a tetrad simply by applying the rotation (1.9), (1.12)] and writing out the field equations in these coordinates; in fact this is the method by which Farnsworth obtained the dust solutions. These solutions are special cases of those classified by Stewart and Ellis [13], and are the only expanding, tilted spatially homogeneous cosmologies admitting symmetry groups of dimension greater than 3 .

The second family of universe models with higher symmetry are those in which the 3-surfaces of homogeneity $S(t)$ are surfaces of constant curvature, i.e. in which the Ricci tensor $R^{*}{ }_{a b}$ of these surfaces is isotropic:

$$
R^{*}{ }_{a b}=\frac{1}{3} R^{*} \tilde{h}_{a b}, \quad R^{*} \equiv R_{a}^{* a} .
$$

It follows (see [1]) on using a normalized orthonormal basis for which (2.1) holds, that either

or

$$
\begin{gathered}
n_{1}=n_{2}=n_{3} \equiv m, \\
n_{1}=0, n_{2}=n_{3} \equiv n \neq 0 .
\end{gathered}
$$


In the first case, one must have $a \neq 0$ (or the $(0 v)$ equations imply that $\tilde{q}_{v}=0$ ) and so by (2.12), $m=0$. Thus when (4.5a) is satisfied, the only tilted solutions are type $\mathrm{V}$ universe models. In fact whenever the group is type $\mathrm{V}, R^{*}{ }_{a b}$ is necessarily isotropic and $R^{*}<0$.

In the second case when $(4.5 \mathrm{~b})$ holds, the Jacobi identities imply $\tilde{\sigma}_{12}=\tilde{\Omega}_{3}, \tilde{\sigma}_{13}=-\tilde{\Omega}_{2}, \tilde{\sigma}_{23}=0, \tilde{\theta}_{2}=\tilde{\theta}_{3}$. The (23) field equations show that

$$
\tilde{\pi}_{23}=2 \tilde{\sigma}_{12} \tilde{\sigma}_{13} .
$$

The tetrad can be rotated in the $\left(\tilde{\boldsymbol{e}}_{2}, \tilde{\boldsymbol{e}}_{3}\right)$ plane while still preserving conditions (2.21) and (4.5b); we choose such a rotation to set $\tilde{\sigma}_{13}=0$ $\Rightarrow \tilde{\Omega}_{2}=0$. Now (4.6) shows that at least one of $\tilde{q}_{2}, \tilde{q}_{3}$ must vanish. If $\tilde{q}_{3}=0$, then the (03) equation shows that $\tilde{\sigma}_{12}=0($ as $n \neq 0)$, and so $\tilde{q}_{2}=0$ by the (02) equation; but then the space-time is an L.R.S. space admitting a group of type $\mathrm{V}$, and so can be regarded as a special case of (4.5a). We therefore assume $\tilde{q}_{3} \neq 0$; then $\tilde{q}_{2}=0$, and $\tilde{\pi}_{12} \neq 0$ by the (03) equation. The (02) equation shows that $a=0$, the (01) equation that $\tilde{q}_{1}=0$, and the (13) equation that $\tilde{\Omega}_{1}=0$. The basis we have found is such that in addition to ( $4.5 \mathrm{~b})$,

$$
\begin{gathered}
a=0, \tilde{c}_{v}=(0,0,1), \tilde{\theta}_{1} \equiv \alpha, \tilde{\theta}_{2}=\tilde{\theta}_{3} \equiv \gamma, \\
\tilde{\sigma}_{13}=\tilde{\Omega}_{2}=0=\tilde{\sigma}_{23}=\tilde{\Omega}_{1}, \tilde{\sigma}_{12}=\tilde{\Omega}_{3} .
\end{gathered}
$$

The space admits a group of type $\mathrm{VII}_{0}$, and as $R^{*}=0$, it has flat spatial sections. The vector $\tilde{c}_{\alpha}$ is an eigenvector of $n^{\alpha \beta}$ with a non-zero eigenvalue, so $\omega \neq 0$ and the space fulfils the conditions of Theorem 3.5. This is the space found by Demianski and Grischuk [17]; our derivation has shown that, as they asserted, this is the only rotating homogeneous universe with flat spatial surfaces of homogeneity, because any such universe must satisfy (4.4) with $R^{*}=0$.

Theorem 4.2. The tilted universe models in which the surfaces of homogeneity are surfaces of constant curvature, are (a) those in which $R^{*}<0$, admitting a group of type $V$, and additionally admitting groups of type VII $I_{h}$ if they are L.R.S., and (b) the rotating Demianski-Grischuk universes in which $R^{*}=0$, admitting a group of type $V I I_{0}$ and fulfilling the conditions of Theorem 3.5.

Coordinates and the field equations for all type $\mathrm{V}$ universes (in which $R^{*}{ }_{a b}$ is necessarily isotropic) have been given in the previous section. In the case of the type $\mathrm{VII}_{0}$ universe in which (4.7) holds, one can choose coordinates so that the tetrad-coordinate relations $\tilde{\boldsymbol{e}}_{a}=\tilde{e}_{a}^{i} \partial / \partial x^{i}$ take the form

$$
\begin{gathered}
\tilde{\boldsymbol{e}}_{0}=\partial / \partial t, \tilde{\boldsymbol{e}}_{2}=Z(t)^{-1} \partial / \partial x^{2}, \tilde{\boldsymbol{e}}_{3}=Z(t)^{-1} \partial / \partial x^{3}, \\
\tilde{\boldsymbol{e}}_{1}=X(t)^{-1}\left(\partial / \partial x^{1}+\left(N_{0} x^{3}+2 g(t)\right) \partial / \partial x^{2}-N_{0} x^{2} \partial / \partial x^{3}\right),
\end{gathered}
$$


where $n=N_{0} X^{-1}, N_{0}=$ constant; this determines the metric. The fluid flow vector is $\boldsymbol{u}=\cosh \beta \tilde{\boldsymbol{e}}_{0}+\sinh \beta \tilde{\boldsymbol{e}}_{3}$. The conservation equations take the form (3.8) with $Y=Z$; the (22) and (33) field equations imply $2\left(\tilde{\sigma}_{12}\right)^{2}$ $=(\mu+p) \sinh ^{2} \beta$; then the (03), (12) equations show

$$
\tanh \beta=\left(2 W_{0} R_{0} N_{0}^{-2}\right) X Z^{-3}, \quad \tilde{\sigma}_{12}=-\left(W_{0} R_{0} N_{0}^{-1}\right) Z^{-3} .
$$

The second of these equations shows that

$$
g(t)=W_{0} R_{0} N_{0}^{-1} \int X(t) Z(t)^{-4} d t ;
$$

the first implies that one cannot specify an equation of state for the fluid, because $\beta$ is determined by $X$ and $Z$ alone; the conservation equations determine the evolution of $w$ and $r$ and thereby determire an implicit equation of state relating $p$ and $\mu$.

The remaining field equations are the second order equations

$$
\begin{gathered}
\frac{X^{\cdot}}{X}+2 \frac{X^{\cdot}}{X} \frac{Z}{Z}+2 \frac{W_{0}^{2} R_{0}^{2}}{N_{0}^{2} Z^{6}}=\frac{1}{2}(\mu-p)+\Lambda, \\
\frac{Z^{\cdot}}{Z}+\left(\frac{Z}{Z}\right)^{2}+\frac{Z}{Z} \frac{X^{\cdot}}{X}-2 \frac{W_{0}^{2} R_{0}^{2}}{N_{0}^{2} Z^{6}}=\frac{1}{2}(\mu-p)+\Lambda,
\end{gathered}
$$

with the first integral

$$
2 \frac{X}{X} \frac{Z}{Z}+\frac{Z^{\cdot 2}}{Z^{2}}-\frac{W_{0}^{2} R_{0}^{2}}{N_{0}^{2} Z^{6}}=(\mu+p) \cosh ^{2} \beta-p+\Lambda .
$$

Further details of the solutions of these equations are given by Demianski and Grischuk ([17]); it seems, unfortunately, that the equation of state which one finds for the fluid in these models is unrealistic, for it tends to the "stiff matter" state $p=\mu$ near the singularity, and the pressure goes negative and approaches zero from below at large times.

A further class of higher symmetry solutions are the tilted models which are homogeneous space-times, i.e. which are stationary. Then one can find a tetrad basis as before, but in which additionally all the rotation coefficients are constants. All physical quantities (e.g. $\mu, p, \beta)$ must also be constants; solution of the field equations reduces to an algebraic problem, as all one has to do is solve the equations for the constants $\gamma_{b c}^{a}$. We shall not investigate these solutions further here; all the dust solutions are known $([26,27])$, and the perfect fluid solutions could be obtained by the same methods. As the density is constant in these models, they do not expand and so cannot be used as reasonable models of the observed universe. 


\section{Kinematical Restrictions}

We have already considered the restrictions implied by the vanishing of the fluid acceleration [see (1.25) and following] and of the fluid vorticity (see Theorems 3.1 and 3.2). We shall not consider solutions in which the fluid does not expand, as these models cannot produce an isotropic redshift; in fact most, if not all, such models would be included in the homogeneous space-times mentioned in the last section.

Our aim in this section is therefore to see if one can find interesting universe models by putting restrictions on the fluid shear. In particular, one may ask if there are any interesting models with vanishing shear. Gödel's paper [11] suggests such solutions are very restricted; for he showed that shear free type IX dust models cannot both expand and rotate, i.e. for type IX dust universes,

$$
\sigma_{a b}=0 \Rightarrow \theta \omega_{a b}=0 .
$$

This result has been extended by Schücking [28] to all spatially homogeneous dust models, and by Banerji [29] to spatially homogeneous perfect fluid solutions with $p=(\gamma-1) \mu, \gamma=$ constant $\neq 10 / 9$. Here we extend the result to all homogeneous cosmological models in which the matter content is a perfect fluid. In fact we prove a stronger result:

Theorem 5.1. There are no tilted models with vanishing fluid shear.

Now the only non-tilted perfect fluid spatially homogeneous solutions (i.e. solutions obeying statements (1), (2) and (3) of § 1) which are shearfree are the Friedmann or Robertson-Walker universes (this follows because $\omega_{a b}=\dot{u}_{a}=0$ in these models; when in addition $\sigma_{a b}=0$, one has a Robertson-Walker universe [8]). So Theorem 5.1 implies

Theorem 5.2. The only shear-free spatially homogeneous perfect fluid universe models are the Robertson-Walker universe models.

Note that this result is not contradicted by the existence of the Godel [21] universe; for while this is a homogeneous, rotating and shear-free universe, it is not spatially homogeneous in the sense of statement (2) of $\S 1$; for there exist no spatial surfaces of homogeneity $S(t)$ in this universe ${ }^{7}$. By contrast, the Einstein static universe and Oszvath type I model [30] are homogeneous universes which are spatially homogeneous also; the former has vanishing shear, and is a special case of the Robertson-Walker universes, while the latter has non-vanishing rotation and shear.

${ }^{7}$ Given any local spacelike surface $S$, any two points $p, q$ of $S$ can be mapped into each other by some isometry $\Phi_{p q}$; but there will be pairs of points $p, q$ in $S$ for which $\Phi_{p q}(S) \neq S$, i.e. $S$ itself is not invariant under the isometry, and so $S$ cannot be a surface of homogeneity. 
We shall outline the proof of Theorem 5.1; the interested reader is referred elsewhere [32] for details. The proof is in two stages: first a proof that (5.1) holds for tilted models, and then a proof that neither of the alternatives $\sigma_{a b}=\omega_{a b}=0$ or $\sigma_{a b}=\theta=0$ leads to a tilted model. To carry out the proof, we use the normalized fluid basis of $\S 2$ and associated time coordinate (2.13); the field equations needed are given in Appendix A.

The essential point in proving (5.1) is that if $\sigma_{a b}=0$, one can integrate (2.29) and the Jacobi identities up to a quadrature, in terms of the length $l$ defined in $\S 1$ :

$$
\begin{gathered}
\lambda \equiv r \tanh \beta=l^{-1} \lambda_{0}, \quad \omega^{a}=r^{-1} l^{-2} \omega_{0}^{a}, \quad \gamma^{1}{ }_{12}=l^{-1} A_{1}, \quad \gamma^{2}{ }_{12}=l^{-1} A_{2}, \\
\gamma^{1}{ }_{23}=l^{-1} B_{1}, \quad \gamma^{2}{ }_{13}=l^{-1} B_{2}, \\
\gamma_{13}^{1}=\lambda_{0} l^{-1}\left(l^{-1} l_{, 0}+C_{1}\right), \quad \gamma_{23}^{2}=\lambda_{0} l^{-1}\left(l^{-1} l_{, 0}+C_{2}\right)
\end{gathered}
$$

where $\lambda_{0}, \omega_{0}{ }^{a}, A_{1}, A_{2}, B_{1}, B_{2}, C_{1}, C_{2}$ are constants. We use (2.23 a) to set $\omega_{0}{ }^{1}=0$ on $t=t_{0}$, so $\omega_{0}{ }^{1}=0$ and $\omega^{1}=0$ for all $t$. The (23) equation implies

$$
2 \omega_{0}^{2} \omega_{0}^{3}\left(2 \lambda_{0}{ }^{-2}-l^{-2} r^{-2}\right)+\lambda_{0} A_{1}\left(C_{2}-C_{1}\right)-A_{2}\left(B_{1}+B_{2}\right)=0,
$$

so either $\omega_{0}^{2} \omega_{0}^{3}=0$, or $r=r_{0} l^{-1}$ where $r_{0}$ is a constant. The proof is now straightforward if tedious. One simply substitutes into the field equations from (5.2) in the three cases (i) $r=r_{0} l^{-1}, \omega_{0}^{2} \omega_{0}^{3} \neq 0$, (ii) $\omega_{0}{ }^{3}=0, \omega_{0}{ }^{2} \neq 0$, (iii) $\omega_{0}{ }^{2}=0, \omega_{0}{ }^{3} \neq 0$. In all cases the assumption $\omega_{a b} \theta \neq 0$ leads to a contradiction.

To prove there are no tilted models with $\sigma_{a b}=\omega_{a b}=0$, assume $\beta>0$. Then the (03) equation shows that $\theta=$ constant. It follows from (1.22-24) and the equations obtained from them by differentiating and using $\theta=$ constant, that $r$ is linear in $t$. This is incompatible with the remaining field equations unless $\theta=0$; but the only spatially homogeneous perfect fluid solutions with $\sigma_{a b}=\omega_{a b}=\theta=0$ can easily be shown to be the Einstein static universe, which is not tilted.

When $\sigma_{a b}=\theta=0$, the (01) and (03) equations show that $\gamma^{2}{ }_{23}=0$, once one has set $\omega^{1}=0$ by an initial rotation of the basis. The remaining equations imply that $\gamma_{12}^{2}=\gamma^{2}{ }_{13}=0$, and that either (i) $\gamma_{23}^{1}=\gamma_{12}^{1}=0$, or (ii) $\gamma_{23}^{1}=\gamma_{13}^{1}=0$, or (iii) $\gamma_{12}^{1}=\gamma_{13}^{1}=0$. However in each case the constraint equation (C) shows that $\mu+p<0$, contradicting assumption (1.2).

Further restrictions on the shear that might lead to interesting universe models are, for example, that the vorticity vector or the acceleration vector should be a shear eigenvector. In general such conditions are difficult to solve, and we shall restrict our attention to a simpler 
problem, which was again initially raised by Gödel. He stated [11] that, in the case of type IX dust universes,

if $\omega^{a} \neq 0$, at no instant of time can the expansion tensor $\theta_{a b}$

be rotationally symmetric about the vorticity vector $\omega^{a}$.

This statement is concerned with one instant of time only, and so does not concern the propagation equations. We wish to know if $(\mathrm{G})$ applies to other group types, or when the pressure is non-zero; the question to be resolved then, is whether the constraint equations [(A)-(D) in Appendix A] can be solved at any instant so as to violate (G), or not.

Without loss of generality, we can put $\omega^{2}=0$ on the hypersurface $\{t=$ constant $\}$ we are considering. Then the condition that $\theta_{a b}$ be rotationally symmetric about $\omega^{a}$ reduces to

$$
\begin{gathered}
\sigma_{12}=0=\sigma_{23}, \quad \omega^{1} \sigma_{13}=\omega^{3}\left(\theta_{1}-\theta_{2}\right), \\
\left(\omega^{1}\right)^{2} \theta_{3}=\left(\omega^{3}\right)^{2} \theta_{1}+\theta_{2}\left(\left(\omega^{1}\right)^{4}-\left(\omega^{3}\right)^{4}\right) \omega^{-2} .
\end{gathered}
$$

If one puts no condition on $A$, this question has to be decided on the basis of equations (A), (B) and (C) alone; and if further $p \neq 0$, one can in general consider only (A) and (C) as (B) has in it a term $-2 r,{ }_{0} \omega^{1}$ which is in general non-zero.

Theorem 5.3. If $\omega^{a} \neq 0$ is parallel to $c^{a}$ in a tilted model, then Gödel's result $(G)$ is true.

In this case, $\omega^{1}=0 \Rightarrow \theta_{1}=\theta_{2}$. By Theorem 3.5 , the solution is a class A solution; from Appendix B, this implies that in the fluid basis,

$$
\gamma_{13}^{1}+\gamma_{23}^{2}=\lambda\left(\theta_{1}+\theta_{2}\right) / r .
$$

Substituting (5.3) and (5.4) into the constraints, one easily finds that $\mu+p<0$.

This result is the widest form of $(\mathrm{G})$ we have been able to obtain. In fact in general it seems possible to violate $(\mathrm{G})$. Thus consider class $A$ rotating dust solutions with $\omega^{3}=0$ (by Theorem 3.3 , this is only possible in types VIII and $\mathrm{VI}_{0}$ ) and satisfying (5.3), which implies $\theta_{2}=\theta_{3}$. Then one obtains three conditions from the vanishing of $a_{\varepsilon}$ (see Appendix B); constraint (A) shows $\gamma^{1}{ }_{23}+2 \gamma^{2}{ }_{13}=0$; constraint (B) shows $\gamma_{23}^{2}$ $=\frac{2}{3} \lambda^{-1}\left(\theta_{2}-\theta_{1}\right)+\frac{1}{3} \lambda \theta_{2}$, which implies $\gamma_{13}^{1}=\frac{2}{3} \lambda \theta_{2}+\lambda \theta_{1}+\frac{2}{3} \lambda^{-1}\left(\theta_{1}-\theta_{2}\right)$. Now examination of (C) shows that we can satisfy it for $\gamma^{1}{ }_{23} \leqq 0$. Thus (G) does not hold for type VIII or $\mathrm{VI}_{0}$ dust universes, and a fortiori for such solutions with $p>0$. Similarly in type $\mathrm{V}$ dust solutions satisfying (5.3), we also have $n_{\alpha \beta}=0$ which gives a set of conditions on the $\gamma_{b c}^{a}$ (cf. Appendix B); and one finds $\gamma_{23}^{1}=\gamma^{2}{ }_{13}=\gamma^{2}{ }_{12}=\omega^{2}=\omega^{3}=0$, $\theta_{2}=\theta_{3}, \gamma_{13}^{1}-\gamma_{23}^{2}=\lambda\left(\theta_{1}-\theta_{2}\right), \gamma_{12}^{1}=-2 \omega^{1} \lambda^{-1}, \sigma_{\mu v}=0(\mu \neq v)$. The 
constraints $(\mathrm{A})-(\mathrm{C})$ can now be satisfied by suitable choice of the remaining $\gamma$ 's. Thus $(\mathrm{G})$ does not hold in type $\mathrm{V}$ models; and this implies that $(\mathrm{G})$ does not hold in types IV, $\mathrm{VI}_{h}$ or $\mathrm{VII}_{h}$ either, as these types are more general than type $\mathrm{V}$ in the sense that type $\mathrm{V}$ can be obtained from them by specializing particular parameter values (cf. the "specialization diagram" in [3]). Hence if one can find initial data to satisfy (5.3) and keep $\mu+p>0$ in type $\mathrm{V}$, one can certainly do it in these group types where one has more freedom of choice of initial data. Finally we have obtained a set of initial data in a type IX universe with $p \neq 0$ in which (5.3) is satisfied and $\mu+p>0$; this is given by $\gamma^{1}{ }_{23}=-\gamma^{2}{ }_{13}=1=-\omega^{3}$, $\gamma^{1}{ }_{13}=0, \omega^{1}=\frac{1}{2}, \theta_{1}=1, \gamma^{1}{ }_{12}=2 \lambda^{-1} r \omega^{1}, \gamma^{2}{ }_{12}=0, \gamma_{23}^{2}=r^{-1} \lambda\left(\theta_{1}+\theta_{2}\right)$, and $r^{-1} \lambda$ chosen sufficiently small. While we have not investigated type $\mathrm{VII}_{0}$ in detail, we have no reason to believe that $(\mathrm{G})$ holds in this case either when $p>0$.

While one can find initial data in which the expansion is at some instant rotationally symmetric about the vorticity, this restriction by itself does not determine a simple class of rotating solutions for us. It may be that insisting that this condition, or some similar condition, hold at all times would lead to some simple universe models.

\section{Some Simple Class A Solutions}

We should like to have explicitly written out consistent, simple field equations and coordinates for rotating and expanding universes in which the equation of state can be specified arbitrarily. This has been achieved above for class $\mathrm{B}$ universes (the type $\mathrm{V}$ and type IV universes with $n^{\alpha \beta} \tilde{c}_{\beta}=0$ in $\S 3$ ), but not for class A universes (the type II models in $\S 3$ do not rotate, while the Demianski-Grischuk solutions in $\S 4$ could only admit an unrealistic equation of state). We here aim to find a simple set of class A universes satisfying these requirements.

Consider the universes satisfying the requirements of Theorem 3.5. Using a normalized orthonormal basis $(2.21)$, we can choose $\tilde{c}_{\alpha}=(0,0,1)$. The conservation Eq. (1.35) then shows that $\tilde{\Omega}_{1}=-\tilde{\sigma}_{23}, \tilde{\Omega}_{2}=\tilde{\sigma}_{13}$. The (03) equation shows that $\tilde{\sigma}_{12}\left(n_{1}-n_{2}\right) \neq 0$; as one of $n_{1}, n_{2} \neq 0$, we shall assume $n_{2} \neq 0$. If $\tilde{\sigma}_{13} \neq 0$, the Jacobi identities and $(0 v)$ equations show that $n_{1}=n_{3}=0$; but this is a non-rotating type II solution. We therefore consider solutions with

$$
\tilde{\sigma}_{12} n_{2} \neq 0, \tilde{c}_{\alpha}=(0,0,1), \tilde{\sigma}_{23}=0=\tilde{\Omega}_{1}, \tilde{\sigma}_{13}=0=\tilde{\Omega}_{2},
$$

(the latter requirement following from the Jacobi identities). There remains one algebraic Jacobi identity:

$$
\left(n_{1}+n_{2}\right) \tilde{\sigma}_{12}+\left(n_{1}-n_{2}\right) \tilde{\Omega}_{3}=0 .
$$


In order that the solutions rotate, it is necessary that $n_{3} \neq 0$. (The solutions with $n_{3}=0$ are type $\mathrm{VI}_{0}$ and $\mathrm{VII}_{0}$ generalizations of the nonrotating type II solutions in $\S 3$.) The solutions with $n_{1} n_{3} \neq 0$ will be rotating type VIII or IX universes; for simplicity, we consider the simpler type $\mathrm{VI}_{0}$ and $\mathrm{VII}_{0}$ universes with $n_{1}=0$. Then (6.1), (6.2) show:

$$
n_{3} \neq 0, n_{1}=0 \Rightarrow \tilde{\sigma}_{12}=\tilde{\Omega}_{3} .
$$

Clearly these are the simplest universes of the type considered in Theorem 3.5. They are in fact simple generalizations of the DemianskiGrischuk solutions, which are the special case in which $n_{2}=n_{3}$ (which implies $\tilde{\theta}_{2}=\tilde{\theta}_{3}$ ).

With restrictions (6.1), (6.3) one can find coordinates for which

$$
\begin{gathered}
\tilde{\boldsymbol{e}}_{0}=\partial / \partial t, \tilde{\boldsymbol{e}}_{2}=Y(t)^{-1} \partial / \partial x^{2}, \tilde{\boldsymbol{e}}_{3}=Z(t)^{-1} \partial / \partial x^{3}, \\
\tilde{\boldsymbol{e}}_{1}=X(t)^{-1}\left(\partial / \partial x^{1}+\left(N_{2} x^{3}+2 g(t)\right) \partial / \partial x^{2}-N_{3} x^{2} \partial / \partial x^{3}\right),
\end{gathered}
$$

where $n_{2}=(X Z)^{-1} Y N_{2}, n_{3}=(X Y)^{-1} Z N_{3} ; N_{2}, N_{3}$ are constants. The fluid flow vector is $\boldsymbol{u}=\cosh \beta \tilde{\boldsymbol{e}}_{0}+\sinh \beta \tilde{\boldsymbol{e}}_{3}$. The conservation equations are (3.8); the (12) and (03) field equations show that

$$
\tilde{\sigma}_{12}=-W_{0} R_{0}\left(N_{2} Y^{2} Z\right)^{-1} \Rightarrow g=W_{0} R_{0} N_{2}^{-1} \int X\left(Y^{3} Z\right)^{-1} d t .
$$

The remaining field equations are the second order equations

$$
\begin{aligned}
\frac{X^{*}}{X}+\frac{X^{\cdot}}{X} \frac{Y^{\cdot}}{Y}+ & \frac{X^{\cdot}}{X} \frac{Z}{Z}-\frac{1}{2}\left(\frac{N_{2} Y}{X Z}-\frac{N_{3} Z}{X Y}\right)^{2}+\frac{2 W_{0}^{2} R_{0}^{2}}{N_{2}{ }^{2} Y^{4} Z^{2}} \\
& =\frac{1}{2}(\mu-p)+\Lambda, \\
\frac{Y^{*}}{Y}+\frac{Y^{\cdot}}{Y} \frac{Z^{\cdot}}{Z}+ & \frac{Y^{\cdot}}{Y} \frac{X^{\cdot}}{X}+\frac{1}{2}\left(\frac{N_{2} Y}{X Z}\right)^{2}-\frac{1}{2}\left(\frac{N_{3} Z}{X Y}\right)^{2}-\frac{2 W_{0}^{2} R_{0}^{2}}{N_{2}^{2} Y^{4} Z^{2}} \\
& =\frac{1}{2}(\mu-p)+\Lambda, \\
\frac{Z^{*}}{Z}+\frac{Z}{Z} \frac{X^{\cdot}}{X}+ & \frac{Z^{\cdot}}{Z} \frac{Y^{\cdot}}{Y}+\frac{1}{2}\left(\frac{N_{3} Z}{X Y}\right)^{2}-\frac{1}{2}\left(\frac{N_{2} Y}{X Z}\right)^{2} \\
& =\frac{1}{2}(\mu-p)+(\mu+p) \sinh ^{2} \beta+\Lambda
\end{aligned}
$$

with the first integral

$$
\begin{aligned}
\frac{X^{\cdot}}{X} \frac{Y^{\cdot}}{Y} & +\frac{Y^{\cdot}}{Y} \frac{Z^{\cdot}}{Z}+\frac{Z^{\cdot}}{Z} \frac{X^{\cdot}}{X}=(\mu+p) \cosh ^{2} \beta-p+\Lambda \\
& +\frac{1}{4}\left(\frac{N_{2} Y}{X Z}-\frac{N_{3} Z}{X Y}\right)^{2}+\frac{W_{0}^{2} R_{0}^{2}}{N_{2}^{2} Y^{4} Z^{2}} .
\end{aligned}
$$

One can now specify $p(\mu)$ arbitrarily in these rotating solutions $(\omega \neq 0$ when $N_{3} \neq 0$ ); they represent a simple generalization of the Demianski- 
Grischuk solutions, which are obtained from them by letting $Y=Z$ and $N_{2}=N_{3}$ (the extra constraint on the fluid behaviour in that case arises as the consistency condition for $Y^{*} / Y$ and $Z * / Z$ being equal). By the derivation, it is clear that we have obtained all type $V I_{0}$ and $V I I_{0}$ rotating solutions satisfying the condition of Theorem 3.5. Properties of these models have previously been discussed by Ozsvath [14].

\section{Conclusion}

We have considered the geometrical restrictions implied by the condition that a perfect-fluid universe should be spatially homogeneous (in the sense that (1) and (2) of $\S 1$ have been imposed), and concentrated our attention on "tilted" models, i.e. those in which the fluid 4-velocity is not the normal direction to the surface of homogeneity. Our general discussion of these models in $\S 1$ relates the fluid properties to properties of the intrinsically defined direction of tilt in the homogeneous surfaces. An examination of possible tetrads in these spaces $(\S 2)$ enabled us to obtain various relations between geometrically defined quantities and the group types $(\S 3)$ and characterisations of certain higher symmetry models $(\S 4)$. Some properties of the fluid kinematical quantities were also obtained $(\S 5)$, and some simple Class A rotating models obtained in $\S 6$.

Our discussion led us to relatively simple metric forms and field equations for the cases of tilted type II universes, which are necessarily non-rotating; for tilted type V and some type IV universes, which may or may not rotate; and for the Demianski-Grischuk rotating universes with flat spatial sections, and some simple type $\mathrm{VI}_{0}$ and $\mathrm{VII}_{0}$ generalisations. These are probably the simplest tilted models (the Farnsworth type V L.R.S. model being particularly simple); however one might find other simple models by careful consideration of kinematical restrictions. In particular, some Bianchi III models may be relatively simple.

One might feel that some interesting spatially homogeneous universe models had been omitted because the definition (2) of spatial homogeneity was too stringent: the groups of isometries have been restricted to 3-dimensional groups (which are necessarily simply transitive on the surfaces of homogeneity). One could replace condition (2) by the weaker condition.

$\left(2^{\prime}\right)$ space-time is locally invariant under a group of isometries whose surfaces of transitivity are spacelike surfaces $S(t)$,

and consider cosmological models satisfying $(1),\left(2^{\prime}\right)$. Then all of $\S 1$ would still be applicable; in particular one could, as before, separate the models into "tilted" and "non-tilted" universes. 
When $\left(2^{\prime}\right)$ holds the group transforms each surface $S(t)$ into itself, and so acts as a group of isometries in each surface; so the only allowed values are $r=3,4,6$ (cf. $[12,13]$ ). In general the group $G_{r}$ (note that this might not itself be the full group of isometries of the space-time) will possess a subgroup $G_{3}$ which satisfies condition (2). This is trivially true when $r=3$; it is always true when $r=6([1])$; when $r=4$ it is always true except in the case of the Kantowski-Sachs type I universes ([33]). Thus these are the only universes satisfying (2') and not (2). However in them, the Ricci eigenvector is normal to the surfaces $S(t)$ (see Eq. (11 a) in [33]). Hence there are no tilted universes satisfying (1), (2') but not satisfying (1), (2). Thus if we extend the definition of a tilted model $(\S 1)$ to one in which (1), $\left(2^{\prime}\right)$ hold and $\beta>0$, all the results of $\S 3-5$ remain true (but parts of $\S 2$ do not apply to the exceptional universes, cf. $[12,13]$ ), and we do not obtain any further tilted models by broadening (2) to $\left(2^{\prime}\right)$.

The restrictions we have found are based on both kinematics and dynamics; dynamics has entered because we have in each case considered whether the field equations were consistent or not. However we have not given a discussion here of the dynamical evolution of the various tilted models; such discussions can be found for various models, in the papers given as references. There is one aspect of this dynamical evolution which is of particular interest, namely the evolution near any singularity which may occur. We shall return to this question in a later paper; it will turn out that use of the fluid basis of $\S 2$ is particularly helpful in considering some aspects of this question.

We should like to thank M. A.H. MacCallum and R.K. Sachs for advice and discussions, and M. A. H. MacCallum for checking some of the equations used. One of us (A.R.K.) would like to thank the U.K. Science Research Council for a grant.

\section{Appendix A}

Field Equations

(00)

$$
\begin{aligned}
& r \partial_{0} \theta+\theta_{1}^{2}+\theta_{2}^{2}+\theta_{3}^{2}+2 \sigma_{12}^{2}+2 \sigma_{13}^{2}+2 \sigma_{23}^{2}-2(\omega)^{2} \\
& +\lambda\left(\frac{\lambda r_{, 0}}{r}\right)_{, 0}+\frac{\lambda r_{, 0}}{r}\left(-\frac{\lambda r_{, 0}}{r}+\gamma_{13}^{1}+\gamma_{23}^{2}\right)=\Lambda-\frac{1}{2}(\mu+3 p),
\end{aligned}
$$

$$
\lambda \partial_{0}\left(\sigma_{13}+\omega^{2}\right)=\gamma_{\mu 1}^{\lambda} \sigma_{\lambda \mu}-\gamma_{\mu \kappa}^{\mu}\left(\omega_{\kappa 1}+\sigma_{\kappa 1}\right)+\frac{1}{2} \omega_{\mu \lambda} \gamma_{\lambda \mu}^{1}+\frac{2 \lambda r_{, 0} \omega^{2}}{r}
$$

$$
\lambda \partial_{0}\left(\sigma_{23}-\omega^{1}\right)=\gamma_{\mu 2}^{\lambda} \sigma_{\lambda \mu}-\gamma_{\mu \kappa}^{\mu}\left(\omega_{\kappa 2}+\sigma_{\kappa 2}\right)+\frac{1}{2} \omega_{\mu \lambda} \gamma_{\lambda \mu}^{2}-\frac{2 \lambda r_{, 0} \omega^{1}}{r}
$$


(03) $-\lambda \partial_{0}\left(\theta_{1}+\theta_{2}\right)=\gamma_{\mu 3}^{\lambda} \sigma_{\lambda \mu}-\gamma_{\mu \kappa}^{\mu}\left(\omega_{\kappa 3}+\sigma_{\kappa 3}\right)+\frac{1}{2} \omega_{\mu \lambda} \gamma_{\lambda \mu}^{3}$,

(11) $r \partial_{0} \theta_{1}-\lambda \partial_{0} \gamma_{13}^{1}+\theta \theta_{1}+\frac{\lambda r_{, 0} \gamma_{13}^{1}}{r}+\gamma_{\mu \nu}^{\mu} \gamma_{\nu 1}^{1}$

$$
\begin{aligned}
& -2\left[\sigma_{12}^{2}+\sigma_{13}^{2}-\left(\omega^{2}\right)^{2}-\left(\omega^{3}\right)^{2}\right]-\left(\gamma_{12}^{2}\right)^{2}-\frac{4 r^{2}\left(\omega^{2}\right)^{2}}{\lambda^{2}}-\frac{2 r^{2}\left(\omega^{3}\right)^{2}}{\lambda^{2}} \\
& -\frac{2 \gamma_{13}^{2} r \omega^{3}}{\lambda}+\frac{1}{2}\left[\left(\gamma_{23}^{1}\right)^{2}-\left(\gamma_{13}^{2}\right)^{2}\right]=\Lambda+\frac{1}{2}(\mu-p),
\end{aligned}
$$

(22) $r \partial_{0} \theta_{2}-\lambda \partial_{0} \gamma_{23}^{2}+\theta \theta_{2}+\frac{\lambda r_{, 0} \gamma_{23}^{2}}{r}+\gamma_{\mu \nu}^{\mu} \gamma_{v 2}^{2}$

$$
\begin{aligned}
& +2\left[\left(\omega^{3}-\sigma_{12}\right)^{2}+\left(\omega^{1}\right)^{2}-\sigma_{23}^{2}\right]-\left(\gamma_{12}^{1}\right)^{2}-\frac{4 r^{2}\left(\omega^{1}\right)^{2}}{\lambda^{2}}-\frac{2 r^{2}\left(\omega^{3}\right)^{2}}{\lambda^{2}} \\
& +\frac{2 \gamma_{23}^{1} r \omega^{3}}{\lambda}+\frac{1}{2}\left[\left(\gamma_{13}^{2}\right)^{2}-\left(\gamma_{23}^{1}\right)^{2}\right]=\Lambda+\frac{1}{2}(\mu-p),
\end{aligned}
$$

(33) $r \partial_{0} \theta_{3}+\theta \theta_{3}-\lambda \partial_{0}\left(\gamma_{13}^{1}+\gamma_{23}^{2}\right)+\lambda\left(\frac{\lambda r_{, 0}}{r}\right)_{, 0}-\left(\frac{\lambda r_{10}}{r}\right)^{2}+\gamma_{\mu \nu}^{\mu} \gamma_{v 3}^{3}$

$$
\begin{aligned}
& +2\left[\left(\omega^{2}+\sigma_{13}\right)^{2}+\left(\omega^{1}-\sigma_{23}\right)^{2}\right]-\left(\gamma_{13}^{1}\right)^{2}-\left(\gamma_{23}^{2}\right)^{2}+\frac{2 r^{2}\left(\omega^{3}\right)^{2}}{\lambda^{2}} \\
& -\frac{1}{2}\left(\gamma_{23}^{1}+\gamma_{13}^{2}\right)^{2}=\Lambda+\frac{1}{2}(\mu-p) .
\end{aligned}
$$

(12) $0=r \partial_{0} \sigma_{12}-\frac{\lambda}{2} \partial_{0}\left(\gamma_{23}^{1}+\gamma_{13}^{2}\right)+\sigma_{12}\left(2 \theta_{1}+\theta_{3}\right)$

$$
\begin{aligned}
& +2 \omega^{3}\left(\theta_{2}-\frac{r \gamma_{23}^{2}}{\lambda}\right)+2 \omega^{1}\left(\frac{r \gamma_{12}^{1}}{\lambda}-\omega^{2}\right)-2 \sigma_{13} \sigma_{23}+\frac{4 r^{2} \omega^{1} \omega^{3}}{\lambda^{2}} \\
& -\gamma_{23}^{1} \gamma_{13}^{1}-\gamma_{13}^{2} \gamma_{23}^{2}+\frac{\lambda r_{, 0}}{2 r}\left(\gamma_{23}^{1}+\gamma_{13}^{2}\right),
\end{aligned}
$$

(23) $0=r \partial_{0}\left(\sigma_{23}-\omega^{1}\right)+\left(\sigma_{23}-\omega^{1}\right)\left(\theta_{1}+2 \theta_{2}\right)+2 \sigma_{12} \sigma_{13}+2 \sigma_{12} \omega^{2}$

$$
-2 \sigma_{13} \omega^{3}+\frac{4 r^{2} \omega^{2} \omega^{3}}{\lambda^{2}}-2 \omega^{2} \omega^{3}+\gamma_{12}^{1}\left(\gamma_{23}^{2}-\gamma_{13}^{1}\right)-\gamma_{12}^{2}\left(\gamma_{23}^{1}+\gamma_{13}^{2}\right),
$$

(31) $0=r \partial_{0}\left(\sigma_{13}+\omega^{2}\right)+\left(\sigma_{13}+\omega^{2}\right)\left(2 \theta_{1}+\theta_{2}\right)+2 \sigma_{23} \omega^{3}+\frac{4 r^{2} \omega^{1} \omega^{3}}{\lambda^{2}}$

$$
-2 \omega^{1} \omega^{3}+\gamma_{12}^{2}\left(\gamma_{23}^{2}-\gamma_{13}^{1}\right)+\gamma_{12}^{1}\left(\gamma_{23}^{1}+\gamma_{13}^{2}\right) .
$$

[To show that $(a b) \equiv(b a)$ one must use the Jacobi Identities below]. [Note also that $\sigma_{\mu \mu}=\theta_{\mu}-\frac{1}{3} \theta$, in $\left.(0 \mu)\right]$. 
Jacobi Identities

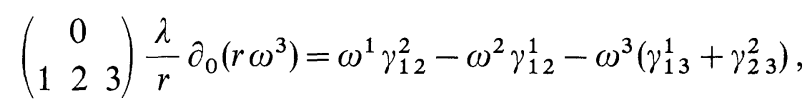

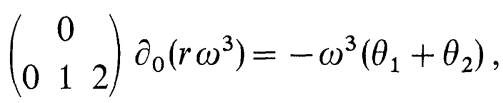

$\left(\begin{array}{lll} & 0 & \\ 0 & 2 & 3\end{array}\right) \partial_{0}\left(r \omega^{1}\right)=-\omega^{1}\left(\theta_{2}+\theta_{3}\right)+2 \sigma_{12} \omega^{2}+2 \sigma_{13} \omega^{3}$,

$\left(\begin{array}{lll} & 0 & \\ 0 & 3 & 1\end{array}\right) \partial_{0}\left(r \omega^{2}\right)=-\omega^{2}\left(\theta_{1}+\theta_{3}\right)+2 \sigma_{23} \omega^{3}$,

$\left(\begin{array}{lll}1 & 2 & 3\end{array}\right)-\frac{\lambda}{2} \partial_{0} \gamma_{12}^{1}=-\theta_{1} \omega^{1}+\frac{r \omega^{1}}{\lambda} \gamma_{13}^{1}+\frac{r \omega^{2}}{\lambda} \gamma_{23}^{1}-2 \sigma_{12} \omega^{2}-2 \sigma_{13} \omega^{3}$ $+\frac{1}{2}\left(\gamma_{12}^{1} \gamma_{23}^{2}-\gamma_{23}^{1} \gamma_{12}^{2}\right)$,

$\left(\begin{array}{lll} & 1 & \\ 0 & 1 & 2\end{array}\right) \partial_{0} \gamma_{12}^{1}=\frac{4 \sigma_{13} \omega^{3}}{\lambda}-\gamma_{12}^{1} \frac{\theta_{2}}{r}+\frac{2 \sigma_{12}}{r} \gamma_{12}^{2}$,

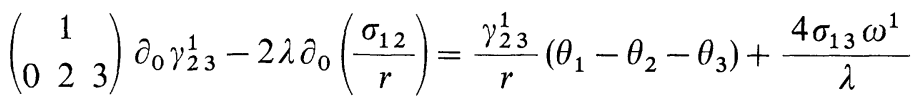
$+\frac{2 \sigma_{13}}{r} \gamma_{12}^{1}+\frac{2 \sigma_{12}}{r}\left(\gamma_{23}^{2}-\gamma_{13}^{1}\right)$

$\left(\begin{array}{lll} & 1 & \\ 0 & 3 & 1\end{array}\right) \lambda \partial_{0}\left(\frac{\theta_{1}}{r}\right)-\partial_{0} \gamma_{13}^{1}=\frac{\theta_{33}}{r} \gamma_{13}^{1}+\frac{4 \sigma_{13}}{r} \omega^{2}+\frac{2 \sigma_{23}}{r} \gamma_{12}^{1}-\frac{2 \sigma_{12}}{r} \gamma_{13}^{2}$,

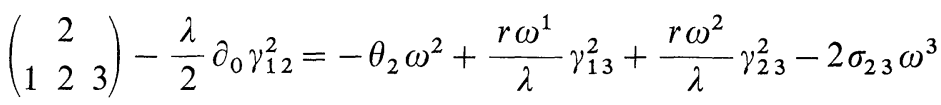

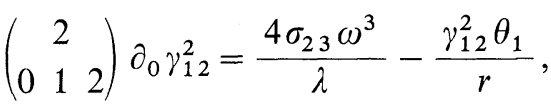

$+\frac{1}{2}\left(\gamma_{12}^{2} \gamma_{13}^{1}-\gamma_{13}^{2} \gamma_{12}^{1}\right)$

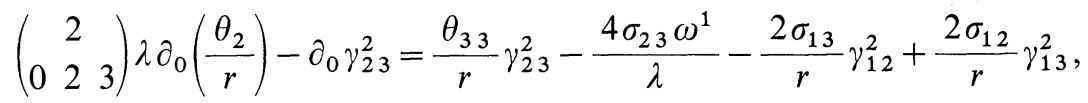

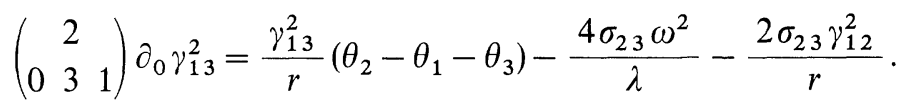

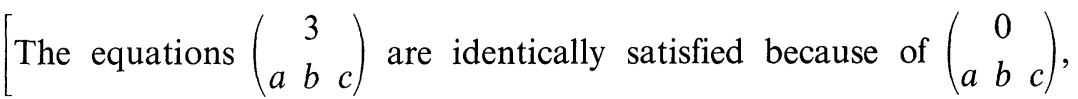
using $\lambda, 0=-\frac{\theta_{3}}{r} \lambda$. 
The four first integrals of the field equations are

$$
\begin{aligned}
\text { (A): } & \frac{r}{\lambda}\left\{\gamma_{\mu 1}^{\lambda} \sigma_{\lambda \mu}-\gamma_{\mu \kappa}^{\mu}\left(\omega_{\kappa 1}+\sigma_{\kappa 1}\right)+\frac{1}{2} \omega_{\mu \lambda} \gamma_{\lambda \mu}^{1}+\frac{2 \lambda r_{, 0}}{r} \omega^{2}\right\} \\
& +\left(\sigma_{13}+\omega^{2}\right)\left(2 \theta_{1}+\theta_{2}\right)+2 \sigma_{23} \omega^{3}+\frac{4 r^{2} \omega^{1} \omega^{3}}{\lambda^{2}}-2 \omega^{1} \omega^{3} \\
& +\gamma_{12}^{2}\left(\gamma_{23}^{2}-\gamma_{13}^{1}\right)+\gamma_{12}^{1}\left(\gamma_{23}^{1}+\gamma_{13}^{2}\right)=0,
\end{aligned}
$$

(B): $\frac{r}{\lambda}\left\{\gamma_{\mu 2}^{\lambda}-\gamma_{\mu \kappa}^{\mu}\left(\omega_{\kappa 2}+\sigma_{\kappa 2}\right)+\frac{1}{2} \omega_{\mu \lambda} \gamma_{\lambda \mu}^{2}-\frac{2 r_{, 0} \omega^{1}}{\lambda}\right\}$

$+\left(\sigma_{23}-\omega^{1}\right)\left(2 \theta_{2}+\theta_{1}\right)+2 \sigma_{12} \sigma_{13}+2 \sigma_{12} \omega^{2}-2 \sigma_{13} \omega^{3}+\frac{4 r^{2} \omega^{2} \omega^{3}}{\lambda^{2}}$

$-2 \omega^{2} \omega^{3}+\gamma_{12}^{1}\left(\gamma_{23}^{2}-\gamma_{13}^{1}\right)-\gamma_{12}^{2}\left(\gamma_{23}^{1}+\gamma_{13}^{2}\right)=0$,

(C): $\left(\frac{\lambda}{r}+\frac{r}{\lambda}\right)\left\{\gamma_{\mu 3}^{\lambda} \sigma_{\lambda \mu}-\gamma_{\mu \kappa}^{\mu}\left(\omega_{\kappa 3}+\sigma_{\kappa 3}\right)+\frac{1}{2} \omega_{\mu \lambda} \gamma_{\lambda \mu}^{3}\right\}$

$-\frac{\lambda r_{, 0}}{r}\left\{\gamma_{13}^{1}+\gamma_{23}^{2}-\frac{\lambda}{r}\left(\theta_{1}+\theta_{2}\right)\right\}-\theta_{1}{ }^{2}-\theta_{2}{ }^{2}+\theta_{3}\left(\theta_{1}+\theta_{2}\right)$

$-2{\sigma_{12}}^{2}-2{\sigma_{13}}^{2}-2 \sigma_{23}{ }^{3}+2(\omega)^{2}+\gamma_{\mu \nu}^{\mu} \gamma_{\nu 3}^{3}+\frac{\lambda \theta_{3}}{r}\left(\gamma_{13}^{1}+\gamma_{23}^{2}\right)$

$+2\left[\left(\omega^{2}+\sigma_{13}\right)^{2}+\left(\omega^{1}-\sigma_{23}\right)^{2}\right]-\left(\gamma_{13}^{1}\right)^{2}-\left(\gamma_{23}^{2}\right)^{2}+\frac{2 r^{2}\left(\omega^{3}\right)^{2}}{\lambda^{2}}$

$-\frac{1}{2}\left(\gamma_{23}^{1}+\gamma_{13}^{2}\right)^{2}+4 \sigma_{13} \omega^{2}-4 \sigma_{23} \omega^{1}+\frac{2 \lambda \sigma_{23}}{r} \gamma_{12}^{1}-\frac{2 \lambda \sigma_{13}}{r} \gamma_{12}^{2}$

$=\mu+p$,

(D): $2\left(\theta_{1} \theta_{2}+\theta_{2} \theta_{3}+\theta_{3} \theta_{1}\right)+6(\omega)^{2}-2{\sigma_{12}}^{2}-2{\sigma_{23}}^{2}-2{\sigma_{13}}^{2}$

$$
\begin{aligned}
& -4 \omega^{1} \sigma_{23}+4 \omega^{2} \sigma_{13}-4 \omega^{3} \sigma_{12}+\frac{2 \lambda^{2} r_{, 0}}{r^{2}}\left(\theta_{1}+\theta_{2}\right) \\
& +\frac{2 \lambda}{r}\left\{\theta_{3}\left(\gamma_{13}^{1}+\gamma_{23}^{2}\right)+\gamma_{\mu 3}^{\lambda} \sigma_{\lambda \mu}-\gamma_{\mu \kappa}^{\mu}\left(\omega_{\kappa 3}+\sigma_{\kappa 3}\right)+\frac{1}{2} \omega_{\mu \lambda} \gamma_{\lambda \mu}^{3}\right\} \\
& -\frac{2 r^{2}}{\lambda^{2}}\left[2\left(\omega^{1}\right)^{2}+2\left(\omega^{2}\right)^{2}+\left(\omega^{3}\right)^{2}\right]-\left(\gamma_{12}^{1}\right)^{2}-\left(\gamma_{12}^{2}\right)^{2}-\left(\gamma_{13}^{1}\right)^{2}-\left(\gamma_{23}^{2}\right)^{2} \\
& +\gamma_{\mu \nu}^{\mu} \gamma_{\lambda \nu}^{\lambda}-\frac{1}{2}\left(\gamma_{23}^{1}+\gamma_{13}^{2}\right)^{2}+8 \sigma_{13} \omega^{2}-8 \sigma_{23} \omega^{1} \\
& +\frac{4 \lambda}{r}\left(\sigma_{23} \gamma_{12}^{1}-\sigma_{13} \gamma_{12}^{2}\right)+\frac{2 r \omega^{3}}{\lambda}\left(\gamma_{23}^{1}-\gamma_{13}^{2}\right) \\
& =2 \Lambda+2 \mu .
\end{aligned}
$$




\section{Appendix B}

$$
\begin{gathered}
{ }_{B}=\left(\begin{array}{ccc}
\kappa \gamma_{23}^{1}-\frac{2 \kappa \lambda \sigma_{12}}{r} & \frac{\kappa}{2}\left(\gamma_{23}^{2}-\gamma_{13}^{1}\right)+\frac{\lambda \kappa}{2 r}\left(\theta_{1}-\theta_{2}\right) & \frac{1}{2} \gamma_{12}^{1}+\frac{r \omega^{1}}{\lambda} \\
\frac{\kappa}{2}\left(\gamma_{23}^{2}-\gamma_{13}^{1}\right)+\frac{\lambda \kappa}{2 r}\left(\theta_{1}-\theta_{2}\right) & -\kappa \gamma_{13}^{2} & \frac{1}{2} \gamma_{12}^{2}+\frac{r \omega^{2}}{\lambda} \\
\frac{1}{2} \gamma_{12}^{1}+\frac{r \omega^{1}}{\lambda} & \frac{1}{2} \gamma_{12}^{2}+\frac{r \omega^{2}}{\lambda} & \frac{2 r \omega^{3}}{\kappa \lambda}
\end{array}\right) \\
a^{\beta}=\left(\frac{1}{2} \gamma_{12}^{2}-\frac{r \omega^{2}}{\lambda},-\frac{1}{2} \gamma_{12}^{1}+\frac{r \omega^{1}}{\lambda},-\frac{\kappa}{2}\left(\gamma_{13}^{1}+\gamma_{23}^{2}\right)+\frac{\lambda \kappa}{2 r}\left(\theta_{1}+\theta_{2}\right)\right)
\end{gathered}
$$

when $\kappa=\cosh \beta$.

\section{References}

1. Ellis, G.F.R., MacCallum, M.A.H.: Commun. math. Phys. 12, 108 (1969).

2. MacCallum, M.A.H., Ellis, G.F. R.: Commun. math. Phys. 19, 31 (1970).

3. MacCallum, M.A.H.: Commun. math. Phys. 20, 57 (1971).

4. Heckmann, O., Schücking, E.: Relativistic Cosmology, in Gravitation, ed. L. Witten 438. New York: Wiley 1962.

5. Estabrook,F.B., Wahlquist, H.D., Behr, C.G.: J. Math. Phys. 9, 497 (1968).

6. Ozsváth, I.: J. Math. Phys. 11, 2860 (1970).

7. Ehlers, J.: Akad. Wiss. u. Lit. (Mainz), Abh. Math. Nat. Kl. No 11 (1961).

8. Ellis, G.F.R.: Relativistic Cosmology, in General Relativity and Cosmology, ed. R. K. Sachs, XLVII Enrico Fermi Summer School proceedings 104. New York: Academic Press 1971.

9. Schouten,J.A.: Ricci Calculus. Berlin-Göttingen-Heidelberg: Springer 1954.

10. Schmidt, B. G.: article in proceedings of Banff Summer School, 1972.

11. Gödel, K.: Proc. Int. Math. Cong. (Camb., Mass) 1, 175, (Am. Math. Soc. Providence), (1952).

12. Ellis, G.F.R.: J. Math. Phys. 8, 1171 (1967).

13. Stewart, J.M., Ellis, G. F. R.: J. Math. Phys. 9, 1072 (1968).

14. Ozsváth,I.: J. Math. Phys. 12, 1078 (1971).

15. Collins, C. B., Hawking, S. W.: Astrophys. J. 180, 317 (1973).

16. Farnsworth, D.L.: J. Math. Phys. 8, 2315 (1967).

17. Demianski, M., Grischuk, L.P.: Commun. math. Phys. 25, 233 (1972).

18. Hawking, S. W.: Mon. Not. Roy. Ast. Soc. 142, 129 (1969).

19. Matzner, R. A.: Astrophys. J. 157, 1085 (1969).

20. Shepley, L.: Ph. D. Thesis, Princeton University (1965); Proc. Nat. Acad. Sci. 52, 1403 (1964). - Matzner, R.A., Shepley,L.C., Warren,J.A.: Ann. Phys. (N.Y.) 57, 401 (1970).

21. Matzner, R.A.: J. Math. Phys. 11, 2432 (1970); 13, 931 (1972); Ann. Phys. (N.Y.) 65, 438, 482 (1971). - Matzner, R.A., Chitre,D.M.: Commun. math. Phys. 22, 173 (1971). - Matzner, R. A., Misner, C. W.: Astrophys. J. 171, 415, 433 (1972).

22. Ryan, M.P.: J. Math. Phys. 10, 1724 (1969); Ann. Phys. 65, 506 (1971); 68, 541 (1971); 70, 301 (1972).

23. Belinskii,V.A., Khalatnikov,I.M., Lifshitz,E.M.: Z.E.T.F. 60, 1969 (1971). Grischuk, L.P., Doroshkevich, A. G., Lukash, V.N.: Z.E.T.F. 61, 3 (1971). 
24. Grischuk, L.P., Doroshkevich,A.G., Novikov,I.D.: Z.E.T.F. 55, 2281 (1968); (Sov. Phys. J.E.T.P. 28, 1214 (1969)).

25. Ruzmaikina, T.V., Ruzmaikin,A.A.: Z.E.T.F. 56, 1742 (1969); (Sov. Phys. J.E.T.P. 29, 934 (1969)).

26. Ozsváth, I.: J. Math. Phys. 6, 590 (1965).

27. Farnsworth, D.L., Kerr, R.P.: J. Math. Phys. 7, 1625 (1966).

28. Schücking, E.: Naturwissen. 19, 507 (1957).

29. Banerji, S.: Prog. Theor. Phys. 39, 365 (1968).

30. Ozsváth,I., Schücking,E.: Ann. Phys. (N.Y.), 55, 166 (1969).

31. Gödel, K.: Rev. Mod. Phys. 21, 447 (1949).

32. King, A.R.: Ph. D. Thesis, University of Cambridge, in preparation.

33. Kantowski, R., Sachs, R. K.: J. Math. Phys. 7, 443 (1966).

34. Taub, A.H.: Proceedings of the 1967 Colloque on «Fluids et champ gravitationnel en relativité générale», No. 170, 57, Paris: Centre National de la Recherche Scientifique (1969).

35. MacCallum, M.A.H., Taub, A.H.: Commun. math. Phys. 25, 173 (1972).

\author{
A. R. King \\ G. F. R. Ellis \\ Department of Applied Mathematics and \\ Theoretical Physics \\ University of Cambridge \\ Silver Street \\ Cambridge CB3 9EW/U.K.
}

